Anna Blaszczok (D)

\title{
Distances of elements in valued field extensions
}

\author{
Received: 7 January 2017 / Accepted: 14 December 2018 /
}

Published online: 21 December 2018

\begin{abstract}
We develop a modification of a notion of distance of an element in a valued field extension introduced by F.-V. Kuhlmann. We show that the new notion preserves the main properties of the distance and at the same time gives more complete information about a valued field extension. We study valued field extensions of prime degree to show the relation between the distances of the elements and the corresponding extensions of value groups and residue fields. In connection with questions related to defect extensions of valued function fields of positive characteristic, we present constructions of defect extensions of rational function fields $K(x, y) \mid K$ generated by elements of various distances from $K(x, y)$. In particular, we construct dependent Artin-Schreier defect extensions of $K(x, y)$ of various distances.
\end{abstract}

\section{Introduction}

In this paper, we consider fields equipped with (Krull) valuations. A valued field will be denoted by $(K, v)$, its value group by $v K$, and its residue field by $K v$. By a valued field extension $(L \mid K, v)$ we mean a field extension $L \mid K$, where $v$ is a valuation of $L$ and $K$ is equipped with the restriction of $v$. For the basic facts about valued fields and their extensions, we refer the reader to $[8,9,21,26,27]$. The algebraic closure of a given field $K$ will be denoted by $\tilde{K}$ and the divisible hull of an abelian group $\Gamma$ will be denoted by $\tilde{\Gamma}$.

If $(L \mid K, v)$ is a valued field extension, then every element $z \in L$ induces a cut of the divisible hull $\widetilde{v K}$ of the value group of $K$ in the following way. The lower cut set is the smallest initial segment of $\widetilde{v K}$ which contains the set $\{v(z-c) \mid c \in K\} \cap v K$. Kuhlmann introduced this cut in [17] and called it the distance of $z$ from $K$, denoted by dist $(z, K)$. In her thesis [1] the author modified this definition, replacing the set $\{v(z-c) \mid c \in K\} \cap v K$ by $\{v(z-c) \mid c \in K\} \cap \widetilde{v K}$. The cut in $\widetilde{v K}$ having as its lower cut set the smallest initial segment of $\widetilde{v K}$ containing $\{v(z-c) \mid c \in K\} \cap \widetilde{v K}$ is denoted by dist $\widetilde{K}(z, K)$. The motivation for this modification is as follows. The lower cut set of dist $(z, K)$ may not contain complete information about the set $v(z-K)$. This appears when the set is not contained in the value group $v K$ of

I wish to thank Franz-Viktor Kuhlmann for inspiring discussions and suggestions.

A. Blaszczok $(\varangle)$ : Institute of Mathematics, University of Silesia, Bankowa 14, 40-007 Katowice, Poland. e-mail: anna.blaszczok@us.edu.pl

Mathematics Subject Classification: 12J10 - 12J25

https://doi.org/10.1007/s00229-018-1100-6 
the field $K$. On the other hand, if $z$ is algebraic over $K$, then the lower cut set of dist $\widetilde{K}(z, K)$ contains the whole set $\{v(z-c) \mid c \in K\}$. This gives more complete information about the valued field extension of $K$ generated by $z$.

Take a valued field $(K, v)$. An extension $(L, v)$ of $(K, v)$ is called immediate if the corresponding value group and residue field extensions are trivial, i.e., if $(v L: v K)=[L v: K v]=1$. Take an element $b$ algebraic over $K$ and assume that $v$ extends in a unique way from $K$ to $K(b)$. We say that the element $b$ is weakly immediate over $K$ if the set $\{v(b-c) \mid c \in K\}$ has no maximal element. Theorem 2.1 shows that this holds in particular when the extension $(K(b) \mid K, v)$ is immediate. Take an element $b$ in some valued field extension of $K$. If $b$ is weakly immediate over $K$, then dist $(b, K)=\operatorname{dist}_{\widetilde{K}}(b, K)$. However, the set $v(b-K) \cap v K$ and thus dist $(b, K)$ does not determine whether $b$ is weakly immediate over $K$ or whether the extension $K(b) \mid K$ is immediate. An important problem connected with valued field extensions is to understand the structure of so-called defect extensions (for the definition see Sect. 2.2). The existence of such extensions is one of the main obstacles for the solution of many open problems in valuation theory. Distances turned out to be an important tool for the study of the structure of defect extensions of valued fields of positive characteristic (cf. [17] and [2]). F.-V. Kuhlmann and O. Piltant in their joint work [22] relate defect extensions with higher ramification groups, in connection with the local uniformization problem. They use distances to describe the relation adopting the modification of the definition of the distance mentioned above. In the study of valuations of rational function fields presented in this paper, which continues the work of S. D. Cutkosky and O. Piltant ([7]), the problem of an upper bound of the number of essentially distinct distances of immediate and defect extensions came up. In the preliminary version of [22] the authors used the old notion of distance. Since this notion does not determine whether an element is immediate over a given valued field, this turned out to be not enough to solve the problem of an upper bound of the number of distances. One of the aim of introducing the new notion of distance was to develop a new, stronger tool which would help to solve the mentioned problem.

In Sect. 2 we recall basic facts about dist $(z, K)$. Then in Sect. 3 we describe the basic properties if dist $\widetilde{K}(z, K)$ and show that many of the important properties of dist $(z, K)$ remain true for $\operatorname{dist}_{\tilde{K}}(z, K)$.

In Sect. 4 we apply the properties of distances to the case of extensions $(K(a) \mid K, v)$ of prime degree. We first show that dist $\widetilde{K}(a, K)$ encodes information about the value and the residue field extension of such a valued field extension (cf. Lemma 4.1 and Proposition 4.2). We then describe the possible distances of elements of extensions of prime degree. Whereas in the case of defectless extensions the possible distances of elements are tightly connected with the value group extension (cf. Proposition 4.5), for defect extensions the situation is much more complicated. In Proposition 4.6 we reduce the problem to the case of distances of purely inseparable weakly immediate elements. In Theorem 4.7 we show that already in the case of valuations of a rational function field $K(x, y) \mid K$ of positive characteristic which are trivial on $K$, we can obtain various forms of distances of weakly immediate purely inseparable elements. More precisely, for a given nondiscrete and non- $p$-divisible subgroup $\Gamma$ of $\mathbb{Q}$ we can construct a valuation of 
$K(x, y)$ trivial on $K$ and such that $y^{1 / p}$ is an element weakly immediate over $K(x, y)$ and its distance is a cut in $\mathbb{Q}$ induced by any given real number or is of the form $(\mathbb{Q}, \emptyset)$.

Describing valuations on rational function fields is one of the important questions in valuation theory with applications to algebraic geometry. The construction of valuations of a rational function field with a given value group was studied already in [25]. Since then, constructions and properties of valued rational function fields were studied by many authors. A classification of all possible extensions of a valuation from a ground field $\mathrm{K}$ to a rational function field $K\left(x_{1}, \ldots, x_{n}\right)$ was studied in particular in [15] and [4]. The authors determine there which value groups and residue fields can appear for such extensions and show how to construct them. The proof of Theorem 4.7 of the present paper shows in particular a possible construction of a valuation of a rational function field $K(x, y) \mid K$ with residue field $K$ and a given value group $\Gamma$. However, in connection with questions related to defect extensions, the main aim of the result is to present a construction of defect extensions of $K(x, y)$ generated by elements of various distances from $K(x, y)$.

Current research connected with local uniformization in positive characteristic shows the importance of a better understanding of the structure of defect extensions of function fields. In particular, we are interested in the construction of ArtinSchreier defect extensions of rational function fields of characteristic $p>0$ and in the distances of their generators. The problem of constructing towers of ArtinSchreier defect extensions in the case of a $p$-divisible value groups was studied in [2]. In the last part of Sect. 4 we use Theorem 4.7 to give a construction of Artin-Schreier defect extensions of the rational function field $K(x, y)$ such that the distance dist $(\vartheta, K(x, y))$ of the Artin-Schreier generator $\vartheta$ from $K(x, y)$ is a given negative real number. This in particular gives constructions of Artin-Schreier defect extensions of rational function fields in two variables in the case of non- $p$ divisible value groups.

\section{Preliminaries}

\subsection{Cuts and distances}

We recall basic notions and facts connected with cuts of ordered abelian groups and the notion of distance of elements of valued field extensions. For the details see Section 2.3 of [17] and Section 3 of [23].

Take a totally ordered set $(T,<)$. For a nonempty subset $U$ of $T$ and an element $a \in T$ we will write $U<a$ if $u<a$ for every $u \in U$. A set $\Lambda^{L} \subseteq T$ is called an initial segment of $T$ if for each $\alpha \in \Lambda^{L}$ every $\beta<\alpha$ also lies in $\Lambda^{L}$. A pair $\left(\Lambda^{L}, \Lambda^{R}\right)$ of subsets of $T$ is called a cut in $T$ if $\Lambda^{L}$ is an initial segment of $T$ and $\Lambda^{R}=T \backslash \Lambda^{L}$. To compare cuts in $(T,<)$ we will use the lower cut sets comparison. That is, for two cuts $\Lambda_{1}=\left(\Lambda_{1}^{L}, \Lambda_{1}^{R}\right), \Lambda_{2}=\left(\Lambda_{2}^{L}, \Lambda_{2}^{R}\right)$ in $T$ we will write $\Lambda_{1}<\Lambda_{2}$ if $\Lambda_{1}^{L} \varsubsetneqq \Lambda_{2}^{L}$, and thus $\Lambda_{1} \leq \Lambda_{2}$ if $\Lambda_{1}^{L} \subseteq \Lambda_{2}^{L}$. 
Take a totally ordered set $(T,<)$. With an element $s \in T$ we can connect two cuts:

$$
\begin{aligned}
& s^{-}:=(\{t \in T \mid t<s\},\{t \in T \mid t \geq s\}), \\
& s^{+}:=(\{t \in T \mid t \leq s\},\{t \in T \mid t>s\}) .
\end{aligned}
$$

We identify the element $s$ with $s^{+}$. Thus, for a cut $\Lambda=\left(\Lambda^{L}, \Lambda^{R}\right)$ in $T$ and an element $s \in T$ the inequality $\Lambda<s$ means that for every $t \in \Lambda^{L}$ we have $t<s$.

Similarly, for any subset $M$ of $T$ we define $M^{+}$to be the cut $\left(\Lambda^{L}, \Lambda^{R}\right)$ in $T$ such that $\Lambda^{L}$ is the least initial segment containing $M$, that is,

$$
M^{+}=(\{t \in T \mid \exists m \in M: t \leq m\},\{t \in T \mid t>M\}) .
$$

We denote by $M^{-}$the cut $\left(\Lambda^{L}, \Lambda^{R}\right)$ in $T$ such that $\Lambda^{L}$ is the largest initial segment disjoint with $M$, i.e.,

$$
M^{-}=(\{t \in T \mid t<M\},\{t \in T \mid \exists m \in M: t \geq m\}) .
$$

Take a cut $\Lambda=\left(\Lambda^{L}, \Lambda^{R}\right)$ in an ordered abelian group $\Gamma$. For any element $\alpha \in \Gamma$ we set

$$
\alpha+\Lambda:=\left(\alpha+\Lambda^{L}, \alpha+\Lambda^{R}\right) .
$$

For every extension $(L \mid K, v)$ of valued fields and $z \in L$ we define

$$
v(z-K):=\{v(z-c) \mid c \in K\} .
$$

The set $v(z-K) \cap v K$ is an initial segment of $v K$ (cf. Lemma 2.16 of [17]) and thus the lower cut set of a cut in $v K$. However, it is more convenient to work with the cut

$$
\operatorname{dist}(z, K)=(v(z-K) \cap v K)^{+} \text {in the divisible hull } \widetilde{v K} \text { of } v K .
$$

As we have mentioned in the Introduction, we call this cut the distance of $z$ from $K$. The lower cut set of dist $(z, K)$ is the smallest initial segment of $\widetilde{v K}$ containing $v(z-K) \cap v K$. If the lower cut set of $\operatorname{dist}(z, K)$ is equal to $\widetilde{v K}$, we will write dist $(z, K)=\infty$. Since dist $(z, K)$ is always a cut in $\widetilde{v K}$, we can compare distances of elements over any algebraic extensions of $(K, v)$, regardless of the respective value group extensions. Indeed, if $(F \mid K, v)$ is an algebraic subextension of $(L \mid K, v)$ then $\widetilde{v F}=\widetilde{v K}$. Thus dist $(z, K)$ and dist $(z, F)$ are cuts in the same group. Since $v(z-K) \cap v K$ is contained in $v(z-F) \cap v F$ we deduce that dist $(z, K) \leq \operatorname{dist}(z, F)$.

If $y$ is another element of $L$ then we define

$$
z \sim_{K} y
$$

to mean that

$$
v(z-y)>\operatorname{dist}(z, K) .
$$

If this holds, then from the definition of distance dist $(z, K)=\operatorname{dist}(y, K)$.

The following theorem gives us important information about the distance of elements in immediate extensions (cf. Theorem 1 of [11] and Theorem 2.19 of [17]). 
Theorem 2.1. If $(L \mid K, v)$ is an immediate extension of valued fields, then for every element $z \in L \backslash K$ the set $v(z-K)$ is contained in $v K$ and has no maximal element. In particular, $v z<\operatorname{dist}(z, K)$.

Take an extension $(L \mid K, v)$ of valued fields and elements $y, z \in L$. If $(K(z) \mid K, v)$ is an immediate extension, then the previous theorem shows that the relation $z \sim_{K} y$ is equivalent to the inequality $v(z-y)>v(z-K)$.

\subsection{Defect and defectless extensions}

A valued field $(K, v)$ is called henselian if the valuation $v$ admits a unique extension to $\widetilde{K}$. A henselization of $(K, v)$ is a minimal henselian field extension of $(K, v)$, in the sense that it admits a valuation preserving embedding over $K$ in every henselian extension of $(K, v)$. Henselizations are unique up to valuation preserving isomorphism over $K$ (cf. Theorem 17.11 of [8]). Thus we will speak of the henselization of $(K, v)$ and denote it by $(K, v)^{h}$ or, if $v$ is fixed, by $K^{h}$. The extension $K^{h} \mid K$ is always immediate and separable-algebraic (cf. [8], Theorem 17.19). Furthermore, if $L$ is any algebraic extension of $K$ and $v$ is a fixed extension from $K$ to the algebraic closure of $K$, then $L^{h}=L \cdot K^{h}$.

Take a valued field $(K, v)$ and a finite extension $L$ of $K$. Assume that the extension of the valuation $v$ from $K$ to $L$ is unique. This is equivalent to the fact that $L$ is $K$-linearly disjoint from the henselization $K^{h}$ of $K$ (cf. Lemma 2.1 of [5]). Then the Lemma of Ostrowski (see [27], Chapter VI, 12, Corollary to Theorem 25) says that

$$
[L: K]=p^{n}(v L: v K)[L v: K v]
$$

with $n \geq 0$ and $p$ the characteristic exponent of $K v$, that is, $p=\operatorname{char} K v$ if it is positive and $p=1$ otherwise. The factor $d(L \mid K, v):=p^{n}$ is called the defect of the extension $(L \mid K, v)$. If it is nontrivial, that is, if $n>0$, then $(L \mid K, v)$ is called a defect extension. If $d(L \mid K, v)=1$, then $(L \mid K, v)$ is called a defectless extension.

Take a finite extension $(L \mid K, v)$ and assume that the valuation of $K$ admits a unique extension to the field $L$. Fix an extension of this valuation to $\tilde{K}$ and denote it again by $v$. Since $K^{h}$ is linearly disjoint from $L$ over $K$ and $K^{h} \mid K$ is an immediate extension, we obtain that

$$
d(L \mid K, v)=d\left(L^{h} \mid K^{h}, v\right) .
$$

Take a defectless extension $(L \mid K, v)$ of henselian fields. Set $e=(v L: v K)$ and $f=[L v: K v]$. Choose elements $\eta_{1}, \ldots, \eta_{e} \in L$ such that $v \eta_{1}, \ldots, v \eta_{e} \in v L$ are representatives of the distinct cosets modulo $v K$. Further, choose $\vartheta_{1}, \ldots, \vartheta_{f}$ in the valuation ring $\mathcal{O}_{L}$ of $(L, v)$ such that $\vartheta_{1} v, \ldots, \vartheta_{f} v$ form a basis of $L v \mid K v$. Without loss of generality we can assume that $\eta_{1}=\vartheta_{1}=1$. Since $L \mid K$ is defectless, $[L: K]=e \cdot f$ and the elements $\eta_{i} \vartheta_{j}, i \leq e$ and $j \leq f$ form a $K$-basis of $L$ (cf., e.g., Lemma 3.2.2 of [9] or Lemma 2.1 of [13]). Such a basis will be called a standard valuation basis. The next lemma (cf. Lemma 2.1 of [23] and Lemma 3.2.2 of [9]) allows us to determine the distance of elements in defectless extensions of henselian fields with the use of a standard valuation basis of the extension. 
Lemma 2.2. With the above assumptions on $(L \mid K, v)$ and on the elements $\eta_{i}$, $i \leq e$ and $\vartheta_{j}, j \leq f$, the set $v(a-K)$ has a maximum for every $a \in L$. More precisely, if

$$
a=\sum_{\substack{i \leq e \\ j \leq f}} c_{i, j} \eta_{i} \vartheta_{j},
$$

then the maximal element of $v(a-K)$ is equal to

$$
v\left(a-c_{1,1}\right)=\min _{(i, j) \neq(1,1)} v\left(c_{i j} \eta_{i} \vartheta_{j}\right)=\min _{(i, j) \neq(1,1)}\left(v c_{i j}+v \eta_{i}\right) .
$$

An important class of extensions satisfying the above assumptions are tame extensions. An algebraic extension $(L \mid K, v)$ of henselian fields is called tame if every finite subextension $E \mid K$ of $L \mid K$ satisfies the following conditions:

(T1) the ramification index $(v E: v K)$ is prime to the characteristic exponent of $K v$,

(T2) the residue field extension $E v \mid K v$ is separable,

(T3) $(E \mid K, v)$ is a defectless extension.

Directly from the above definition it follows that every tame extension of valued fields is separable-algebraic.

Proposition 2.3. Take a henselian field $(K, v)$ and a tame extension $N$ of $K$. Then for any finite extension $L \mid K$,

$$
d(L \mid K, v)=d(L \cdot N \mid N, v) .
$$

For the proof, see [17], Proposition 2.8.

\subsection{Artin-Schreier defect extensions}

In this section we recall a few facts about Artin-Schreier defect extensions of valued fields and their classification presented in detail in [17]. Recall that an Artin-Schreier extension of a field $K$ of positive characteristic $p$ is an extension of degree $p$ generated by a root $\vartheta$ of a polynomial $X^{p}-X-a$ with $a \in K$. In this case, $\vartheta$ is called an Artin-Schreier generator of the extension. Such an extension is always normal and hence Galois. On the other hand, every Galois extension of $K$ of degree $p$ is an Artin-Schreier extension (see, e.g., [24], Chapter VI Galois Theory, 6. Cyclic Extensions).

Towers of Artin-Schreier defect extensions play a central role in the issue of defect extensions. This follows from the fact that every finite separable extension $(L \mid K, v)$ of henselian fields of characteristic $p>0$, lifted up to some finite tame extension $N$ of $K$, becomes a tower of Artin-Schreier extensions (cf. Lemma 2.9 of [17]). Furthermore, by Proposition 2.3 the defect of the lifted extension remains unchanged. Hence, the extension $(L \mid K, v)$ has nontrivial defect if and only if the tower $L . N \mid N$ contains an Artin-Schreier defect extension. 
Throughout the remaining part of this section we assume that $(K, v)$ is a valued field of characteristic $p>0$ and $K(\vartheta) \mid K$ an Artin-Schreier extension with $\vartheta^{p}-\vartheta-a=0$ for some $a \in K$.

Assume that $(K(\vartheta) \mid K, v)$ is a defect extension. Then from the Lemma of Ostrowski it follows that the defect is equal to the degree of the extension. Consequently, the extension is immediate. Furthermore, the valuation $v$ of $K$ admits a unique extension to $K(\vartheta)$. Theorem 2.1 yields that $v(\vartheta-K) \cap v K=v(\vartheta-K)$ has no maximal element.

From Lemma 4.1 of [17] it follows that $\delta:=\operatorname{dist}(\vartheta, K)$ does not depend on the choice of the Artin-Schreier generator $\vartheta$. We call $\delta$ the distance of the ArtinSchreier extension $(K(\vartheta) \mid K, v)$. Lemma 2.30 of [17] implies that $\delta \leq 0^{-}$.

We will distinguish two types of Artin-Schreier defect extensions considering their connection with purely inseparable extensions. Assume that $K(\vartheta) \mid K$ is an Artin-Schreier defect extension. If there is an immediate purely inseparable extension $K(\eta) \mid K$ of degree $p$ such that

$$
\eta \sim_{K} \vartheta
$$

then $K(\vartheta) \mid K$ is called a dependent Artin-Schreier defect extension. Otherwise it is called an independent Artin-Schreier defect extension. The following proposition gives a useful characterization of independent Artin-Schreier defect extensions.

Proposition 2.4. Assume that the extension $(K(\vartheta) \mid K, v)$ has nontrivial defect. Then $K(\vartheta) \mid K$ is an independent Artin-Schreier defect extension if and only if $\operatorname{dist}(\vartheta, K)=H^{-}$for some proper convex subgroup $H$ of $\widetilde{v K}$.

Proof. As we have mentioned, since $\vartheta$ is an Artin-Schreier generator of a defect extension, dist $(\vartheta, K) \leq 0^{-}$. Now the assertion follows directly from Proposition 4.2. and Lemma 2.14 of [17].

We finish the section with considering the form of any separable defect extension of $(K, v)$ of degree $p$.

Lemma 2.5. Assume that $K$ is henselian and $(L \mid K, v)$ is a separable defect extension of degree $p$. Then there is a tame cyclic extension $E \mid K$ of degree dividing $p-1$ such that $(L . E \mid E, v)$ is an Artin-Schreier defect extension. More precisely, there is an element $a \in \tilde{K}$ such that $a^{p-1} \in K$ and $(L(a) \mid K(a), v)$ is an Artin-Schreier defect extension.

Proof. Note that since char $K=p$, the field contains a primitive $(p-1)$-th root of unity. Thus any extension generated by a $(p-1)$-th root of an element of $K$ is cyclic of degree dividing $p-1$ (cf., e.g., Theorem 2.4 of [12]). Moreover, since the degree of the extension divides $p-1$ and $K$ is a henselian field, it is a tame extension. Hence the first assertion of the lemma follows from the second one.

Since $(L \mid K, v)$ is a separable defect extension of degree $p$, Theorem 13 of [16] together with Corollary 2.14 of [20] show that $L=K(\eta)$, where $\eta$ is a root of a polynomial $\mathcal{A}(X)-c$ for some $c \in K$ and an additive polynomial $\mathcal{A}(X) \in K[X]$ 
of degree $p$. Since $L \mid K$ is separable, we obtain that $f(X)=X^{p}-d X-c$ for some $c, d \in K, d \neq 0$. Take $a \in \tilde{K}$ to be a $(p-1)$-th root of $d$ and set $E=K(a)$. As we have noticed, then $(E \mid K, v)$ is a tame extension. Note that

$$
f(X)=X^{p}-a^{p-1} X-c .
$$

Consider the transformation of the polynomial $f$ by setting $X=a Y$ and dividing the polynomial by $a^{p}$. We then obtain the Artin-Schreier polynomial

$$
g(Y)=Y^{p}-Y-\frac{c}{a^{p}} \in E[X] .
$$

Note that $\frac{\eta}{a}$ is a root of $g$. Moreover, $E\left(\frac{\eta}{a}\right)=E(\eta)=L(a)$. Since $E \mid K$ is a tame extension, Proposition 2.3 yields that $d(E(\eta) \mid E, v)=d(K(\eta) \mid K, v)=p$. Thus $E(\eta)=L(a)$ is an Artin-Schreier defect extension of $E=K(a)$.

\subsection{Approximation types}

A very handy tool in studies of immediate extensions of valued fields are approximation types.

Take an extension $(L \mid K, v)$ of valued fields and an element $x \in L$. For every $\alpha \in v K_{\infty}:=v K \cup\{\infty\}$ set

$$
\operatorname{appr}(x, K)_{\alpha}:=\{c \in K \mid v(x-c) \geq \alpha\} .
$$

Note that if $\alpha \leq \beta$, then appr $(x, K)_{\beta} \subseteq \operatorname{appr}(x, K)_{\alpha}$. Furthermore, appr $(x, K)_{\alpha} \neq$ $\emptyset$ if and only if there is $c \in K$ such that $v(x-c) \geq \alpha$. Hence if $x \notin K$, then

$$
S:=\left\{\alpha \in v K_{\infty} \mid \operatorname{appr}(x, K)_{\alpha} \neq \emptyset\right\}=v(x-K) \cap v K=v(x-K) \cap v K_{\infty}
$$

and the set $S$ is an initial segment of $v K$ (cf. Sect. 2.1). If $x \in K$, we obtain that $S=v K_{\infty}=v(x-K) \cap v K_{\infty}$. The set

$$
\operatorname{appr}(x, K):=\left\{\operatorname{appr}(x, K)_{\alpha} \mid \alpha \in v(x-K) \cap v K_{\infty}\right\}
$$

will be called the approximation type of $x$ over $(K, v)$.

Take a valued field extension $(L \mid K, v)$ and $x \in L$. Set $S:=v(x-K) \cap v K_{\infty}$. The approximation type appr $(x, K)$ is called immediate if

$$
\bigcap_{\alpha \in S} \operatorname{appr}(x, K)_{\alpha}=\emptyset .
$$

Note that if $x \in K$, then $S=v K_{\infty}$ and $\bigcap_{\alpha \in S} \operatorname{appr}(x, K)_{\alpha}=\operatorname{appr}(x, K)_{\infty}=\{x\}$. Hence, if the approximation type $\operatorname{appr}(x, K)$ is immediate, then the extension $K(x) \mid K$ is nontrivial.

The next lemma shows the relation between immediate approximation types and immediate extensions of valued fields (cf. Lemma 4.1 of [23]). 
Lemma 2.6. Assume that $(L \mid K, v)$ is an extension of valued fields. If $x \in L$, then the approximation type $\operatorname{appr}(x, K)$ is immediate if and only if the set $v(x-K)$ has no maximal element. If this holds, $v(x-K) \cap v K_{\infty}=v(x-K)$. Furthermore, the extension $(L \mid K, v)$ is immediate if and only iffor every $x \in L \backslash K$ the approximation type $\operatorname{appr}(x, K)$ is immediate.

Take a valued field $(K, v)$ and an element $x$ in some valued field extension $(L, v)$ of $(K, v)$. We say that the approximation type $\operatorname{appr}(x, K)$ fixes the value of $f \in K[X]$ if there is $\alpha \in v(x-K) \cap v K_{\infty}$ such that $v f(c)=v f(d)$ for every elements $c, d \in \operatorname{appr}(x, K)_{\alpha}$. Since $\operatorname{appr}(x, K)_{\beta} \subseteq \operatorname{appr}(x, K)_{\alpha}$, for every $\beta \geq \alpha$, it follows that in this case $v f(c)=v f(d)$ for every $c, d \in \operatorname{appr}(x, K)_{\beta}$ and every $\beta \geq \alpha$. Note that an immediate approximation type $\operatorname{appr}(x, K)$ fixes the value of every linear polynomial in $K[X]$ (cf. Section 5 of [23]). Assume that $\operatorname{appr}(x, K)$ is an immediate approximation type. If $\operatorname{appr}(x, K)$ does not fix the value of some polynomial $f \in K[X]$, then we call it an algebraic approximation type.

Assume that appr $(x, K)$ is algebraic and take a polynomial $f$ of minimal degree whose value is not fixed by appr $(x, K)$. Note that then $f$ is irreducible and can be chosen to be monic. Such a polynomial will be called an associated minimal polynomial for appr $(x, K)$.

Lemma 2.7. Assume that appr $(x, K)$ is an immediate approximation type over $(K, v)$, where $x$ is an element in some valued field extension of $K$.

a) If $x$ is algebraic over $K$, then $\operatorname{appr}(x, K)$ does not fix the value of the minimal polynomial of $x$ over $K$.

b) If $\operatorname{appr}(x, K)$ is an algebraic approximation type and $f$ an associated minimal polynomial for $\operatorname{appr}(x, K)$, then for every $g \in K[X]$ such that $\operatorname{deg} g<\operatorname{deg} f$, the approximation type appr $(g(x), K)$ is also immediate.

For the proof, see Corollary 5.5 and Lemma 8.2 of [23]. Assertion a) of the above lemma yields that if $x$ is algebraic over $K$ then $\operatorname{appr}(x, K)$ is an algebraic approximation type.

\subsection{Power series fields}

We consider now a few properties of an important class of valued fields. For an ordered abelian group $\Gamma$ and a field $k$ take $k\left(\left(x^{\Gamma}\right)\right)$ to be the (generalized) power series field introduced by Hahn in [10]. We introduce the valuation $v$ of $k\left(\left(x^{\Gamma}\right)\right)$ by setting $v f$ to be the minimum of the support of $f$, for every element $f \in k\left(\left(x^{\Gamma}\right)\right)^{\times}$. That is, for every nonzero power series we have

$$
v\left(\sum_{\gamma \in \Gamma} c_{\gamma} x^{\gamma}\right)=\min \left\{\gamma \in \Gamma \mid c_{\gamma} \neq 0\right\} .
$$

This valuation is called the canonical valuation or $\mathbf{x}$-adic valuation of $k\left(\left(x^{\Gamma}\right)\right)$. Directly from the definition of the valuation it follows that $v k\left(\left(x^{\Gamma}\right)\right)=\Gamma$ and 
$k\left(\left(x^{\Gamma}\right)\right) v=k$. Krull in [14] proved that $\left(k\left(\left(x^{\Gamma}\right)\right), v\right)$ is a maximal field, that is, admits no nontrivial immediate extensions. Since the henselization and the completion of a valued field are immediate extensions, every maximal field is henselian and complete. Hence, in particular the power series field $\left(k\left(\left(x^{\Gamma}\right)\right), v\right)$ is henselian and complete.

Take a valued field $(K, v)$ and a subset $A$ of $K$. We say that $A$ is dense in $(K, v)$ if for every $c \in K$ and $\alpha \in v K$ there is an element $a \in A$ such that $v(a-c)>\alpha$.

Lemma 2.8. Take a field $K$, the power series field $K\left(\left(x^{\mathbb{Q}}\right)\right)$ and an element $y \in K\left(\left(x^{\mathbb{Q}}\right)\right)$. Denote by $v$ the restriction of the canonical valuation of the power series field to $K(x, y)$. Then the ring $K\left[x, \frac{1}{x}, y\right]$ is a dense subset of the valued field $(K(x, y), v)$.

Proof. Take any element $u \in K(x, y)$. Then $y=\frac{f}{g}$ for some $f, g \in K\left[x, \frac{1}{x}, y\right]$.

Without loss of generality we can assume that $v g=0$. Indeed, if $v g=\frac{m}{n}$ for some $m \in \mathbb{Z}$ and $n \in \mathbb{N}$, then

$$
u=\frac{x^{-m} f g^{n-1}}{x^{-m} g^{n}},
$$

where $x^{-m} f g^{n-1}, x^{-m} g^{n} \in K\left[x, \frac{1}{x}, y\right]$ and $v x^{-m} g^{n}=0$.

Thus, if $\mathbb{Q}^{\geq 0}$ denotes the set of all nonnegative rational numbers, we obtain that

$$
g=\sum_{\gamma \in \mathbb{Q} \geq 0} a_{\gamma} x^{\gamma}
$$

with $a_{\gamma} \in K$ and $a_{0} \neq 0$. Set $\tilde{f}=a_{0}^{-1} f$ and $h=a_{0}^{-1}\left(a_{0}-g\right)$. Then $\tilde{f}, h \in$ $K\left[x, \frac{1}{x}, y\right]$, the element $h$ is of positive value and $u=\frac{\tilde{f}}{1-h}$.

Take any $\gamma \in \mathbb{Q}$. Since $v h>0$, there is $N \in \mathbb{N}$ such that $v \tilde{f}+(N+1) v h>\gamma$. Thus for

$$
r_{N}:=\tilde{f} \sum_{j=0}^{N} h^{j} \in K\left[x, \frac{1}{x}, y\right]
$$

we obtain that

$$
\begin{aligned}
v\left(u-r_{N}\right) & =v \tilde{f}+v\left(\frac{1}{1-h}-\sum_{j=0}^{N} h^{j}\right) \\
& =v \tilde{f}+v\left(\frac{h^{N+1}}{1-h}\right)=v \tilde{f}+(N+1) v h>\gamma
\end{aligned}
$$

Hence $K\left[x, \frac{1}{x}, y\right]$ is dense in $(K(x, y), v)$. 


\section{Distances of elements in algebraic extensions}

In Sect. 2.1 we have introduced a notion of the distance of an element in a valued field extension. The notion enables us to define and compare distances of elements in any valued field extension $(L, v)$ of a given field $(K, v)$. Nevertheless, since for an element $b \in L$ the lower cut set of dist $(b, K)$ depends only on $v(b-K) \cap v K$, in the case of a nontrivial value group extension we can lose some information about $v(b-K)$.

The next easy observation shows in particular that $v(b-K)$ and $v(b-K) \cap v K$ differ by at most one element.

Lemma 3.1. Assume that $(L \mid K, v)$ is an extension of valued fields and $b \in L \backslash K$.

1) If $v(b-K)$ has no maximal element, then $v(b-K) \subseteq v K$.

2) If $v(b-K)$ admits a maximal element $\alpha$, then $v(b-K) \backslash\{\alpha\} \subseteq v K$ and

$$
v(b-K) \backslash\{\alpha\}=\{\beta \in v K: \beta<\alpha\} .
$$

If moreover $\alpha \in v K$, then $v(b-K) \subseteq v K$ and for any $c, d \in K$ such that $v(b-c)=\alpha=v d$ we have $d^{-1}(b-c) v \notin K v$.

Proof. Take any $c \in K$ such that $v(b-c)$ is not a maximal element of $v(b-K)$. Then there is $d \in K$ such that $v(b-c)<v(b-d)$. Hence

$$
v(b-c)=v(b-c-(b-d))=v(d-c) \in v K .
$$

This proves part 1) and the first assertion of part 2).

For the proof of equality (4) note that since $\alpha$ is the maximal element of $v(b-K)$ and $v(b-K) \backslash\{\alpha\} \subseteq v K$, the inclusion $v(b-K) \backslash\{\alpha\} \subseteq\{\beta \in v K: \beta<\alpha\}$ is obvious. For the proof of the converse, take any $\beta \in v K, \beta<\alpha$. If $\beta=v d$ and $\alpha=v(b-c)$, for some $c, d \in K$, then

$$
\beta=v d=v(b-c+d) \in v(b-K) .
$$

Assume now that $\alpha \in v K$. Then from the first part of assertion 2) we obtain that $v(b-K) \subseteq v K$. Take $c, d \in K$ such that $v(b-c)=\alpha=v d$. Suppose that $d^{-1}(b-c) v \in \bar{K} v$. Take $d^{\prime} \in K$ with $d^{\prime} v=d^{-1}(b-c) v$. Then $\left(d^{-1}(b-c)-d^{\prime}\right) v=$ 0 and thus $v\left(d^{-1}(b-c)-d^{\prime}\right)>0$. It follows that

$$
\alpha=v d<v\left(b-c-d d^{\prime}\right) \in v(b-K),
$$

a contradiction.

Assume that $(K, v)$ is a henselian field with $v K$ densely ordered, and $(L, v)$ a finite extension of $(K, v)$ such that $(v L: v K)=[L: K]$. Then $(L \mid K, v)$ is defectless and from Lemma 2.2 if follows that for every $a \in L \backslash K$ the set $v(a-K)$ admits a maximal element $\alpha_{a} \notin v K$. By the previous lemma,

$$
v(a-K) \cap v K=\left\{\beta \in v K: \beta<\alpha_{a}\right\} .
$$


Since $v K$ is densely ordered and $\alpha_{a} \in \widetilde{v K}$, from the above equality it follows that the set $v(a-K) \cap v K$ has no maximal element.

Take now an immediate algebraic extension $(E, v)$ of $(K, v)$. From Theorem 2.1 we infer that also in this case, for every $a \in E \backslash K$ the set $v(a-K) \cap v K=v(a-K)$ has no maximal element. On the other hand, if for every $a \in E \backslash K$ the set $v(a-K)$ has no maximal element, then by Lemma 2.6, the extension is immediate. The above paragraph shows that the last assertion may not hold if we replace the sets $v(a-K)$ by $v(a-K) \cap v K$.

Note that for every element $b \in \widetilde{K}$, the distance of $b$ from $K$ depends only on $v(b-K) \cap v K$ and not the whole set $v(b-K)$. Hence, as we have seen in the above example, the distances of elements of a given valued field extension may not carry any information whether the considered extension is immediate.

Take a valued field extension $(L \mid K, v)$. Fix an extension of $v$ to the algebraic closure $\widetilde{L}$ of $L$ and denote it again by $v$. For an element $b \in L$ define the distance of $b$ from $K$ over $\widetilde{K}$ to be the following cut in $\widetilde{v K}$ :

$$
\operatorname{dist}_{\widetilde{K}}(b, K):=(v(b-K) \cap \widetilde{v K})^{+}
$$

(cf. the definition of the distance of an element of a valued field from a subset of this field as presented in [23]). As we have mentioned in the Introduction, dist $\widetilde{K}(b, K)$ is the cut in $\widetilde{v K}$ having as its lower cut set the smallest initial segment in $\widetilde{v K}$ containing $v(b-K) \cap v K$. Note that if $b$ is algebraic over $K$ and does not lie in $K$, then $v(b-K) \cap \widetilde{v K}=v(b-K)$. Hence, the lower cut set of dist $\widetilde{K}(b, K)$ contains all of $v(b-K)$ and is therefore the smallest initial segment in $\widetilde{v K}$ containing $v(b-K)$. This notion of distance carries more information about the set $v(b-K)$ than dist $(b, K)$. While the above definition also applies to elements transcendental over $K$, we will focus in our further investigations on the case of algebraic elements. Note that for every $a, b \in \widetilde{K}$ we obtain directly from the definition of the distance that dist $\widetilde{K}(a, K) \leq \operatorname{dist}_{\widetilde{K}}(b, K)$ if and only if $v(a-K) \subseteq v(b-K)$.

As a straightforward consequence of the above definition we obtain the following properties of the distance (cf. Lemma 2.25 of [17]).

Lemma 3.2. Take a valued field $(K, v)$ and an element $b$ algebraic over $K$. Fix an extension of $v$ to $K(b)$. Then for any $c \in K$ we have:

1) $\operatorname{dist}_{\widetilde{K}}(b+c, K)=\operatorname{dist}_{\widetilde{K}}(b, K)$,

2) dist $\widetilde{K}(c b, K)=v c+\operatorname{dist}_{\widetilde{K}}(b, K)$.

Take an element $b \in \widetilde{K}$. As $v(b-K) \cap v K \subseteq v(b-K)$ and dist $(b, K)$, dist $\widetilde{K}(b, K)$ are cuts in the same group $\widetilde{v K}$, from the definitions of the distances it follows that

$$
\operatorname{dist}(b, K) \leq \operatorname{dist}_{\widetilde{K}}(b, K) .
$$

Furthermore, if $v(b-K) \subseteq v K \cup\{\infty\}$, then dist $(b, K)=\operatorname{dist}_{\widetilde{K}}(b, K)$. Hence both definitions of distance coincide in particular in the case of extensions with trivial value group extensions, thus also in the case of immediate extensions. However, using the new notion of distances we can determine if a given algebraic extension is immediate or not. As we have seen, this is not true for the old notion. 
Proposition 3.3. An algebraic extension $(L \mid K, v)$ is immediate if and only if for every element $b \in L \backslash K$ the lower cut set of $\operatorname{dist}_{\widetilde{K}}(b, K)$ has no maximal element.

Proof. This follows directly from Lemma 2.6 and the definition of $\operatorname{dist}_{\widetilde{K}}(b, K)$.

Take a valued field $(K, v)$ and fix an extension of $v$ to $\widetilde{K}$. For $a, b \in \widetilde{K}$ define

$$
a \approx_{K} b
$$

to mean that

$$
v(a-b) \geq \max \left\{\operatorname{dist}_{\widetilde{K}}(a, K), \operatorname{dist}_{\widetilde{K}}(b, K)\right\} .
$$

By inequality (5), in this case also $v(a-b) \geq \max \{\operatorname{dist}(a, K)$, dist $(b, K)\}$. Together with Lemma 3.1 of [23], this yields:

Lemma 3.4. If $a, b \in \widetilde{K}$ are such that $a \approx_{K} b$, then $\operatorname{appr}(a, K)=\operatorname{appr}(b, K)$.

The next lemma gives an important characterization of the relation $\approx_{K}$.

Lemma 3.5. Take elements $a$ and $b$ algebraic over $K$. Then $a \approx_{K} b$ if and only if $v(a-c)=v(b-c)$ for every $c \in K$.

Proof. Assume that $a \approx_{K} b$. Take $c \in K$. Then $v(a-b) \geq v(a-c), v(b-c)$. Therefore,

$$
\begin{aligned}
v(b-c) & \geq \min \{v(b-a), v(a-c)\}=v(a-c) \\
& \geq \min \{v(a-b), v(b-c)\}=v(b-c) .
\end{aligned}
$$

Consequently, $v(b-c)=v(a-c)$.

Suppose now that $v(a-c)=v(b-c)$ for every $c \in K$. Take any $c$ in $K$. Then

$$
\begin{aligned}
v(a-b) & =v(a-c-(b-c)) \geq \min \{v(a-c), v(b-c)\} \\
& =v(a-c)=v(b-c) .
\end{aligned}
$$

By definition of the distance of an element from $K$ over $\widetilde{K}$, we obtain that

$$
v(a-b) \geq \operatorname{dist}_{\widetilde{K}}(a, K)=\operatorname{dist}_{\widetilde{K}}(b, K)
$$

and thus $a \approx_{K} b$.

As direct consequences of the lemma we obtain the following properties, which correspond to the ones that hold for dist $(a, K)$ and the relation $\sim_{K}$ (cf. Lemma 2.17 and 2.25 of [17]).

Corollary 3.6. If $a, b \in \widetilde{K}$ are such that $a \approx_{K} b$, then $\operatorname{dist}_{\widetilde{K}}(a, K)=\operatorname{dist}_{\widetilde{K}}(b, K)$.

Corollary 3.7. Take any elements $a$ and $b$ algebraic over $K$. Then for every $c \in K$ we have:

1) if $a \approx_{K} b$, then $a+c \approx_{K} b+c$;

2) if $a \approx_{K} b$, then $c a \approx_{K} c b$. 
We know already that in the case of an immediate algebraic extension $(K(a) \mid K, v)$, the two notions of distances dist $\widetilde{K}_{(}(a, K)$ and dist $(a, K)$ coincide. The next lemma shows in particular that if $(K(b) \mid K, v)$ is another immediate extension, also the relation $a \approx_{K} b$ can be equivalently replaced by $a \sim_{K} b$.

Proposition 3.8. Take elements $a, b$ algebraic over $K$. If the sets $v(a-K)$ and $v(b-K)$ have no maximal elements, then

$$
a \approx_{K} b \text { if and only if } a \sim_{K} b .
$$

Proof. Note first that since $v(a-K)$ and $v(b-K)$ have no maximal elements, Lemma 3.1 yields that the sets are contained in $v K$. Hence, dist $\widetilde{K}^{(a, K)}=$ $\operatorname{dist}(a, K)$ and dist $\widetilde{K}(b, K)=\operatorname{dist}(b, K)$.

Assume that $a \approx_{K} b$. Then

$$
v(a-b) \geq \max \left\{\operatorname{dist}_{\widetilde{K}}(a, K), \operatorname{dist}_{\widetilde{K}}(b, K)\right\} .
$$

Since the initial segment of $\operatorname{dist}_{\widetilde{K}}(a, K)$ has no maximal element, we obtain that $v(a-b)>\operatorname{dist}_{\tilde{K}}(a, K)=\operatorname{dist}(a, K)$. This gives $a \sim_{K} b$.

Assume now that $a \sim_{K} b$. Then

$$
v(a-b)>\operatorname{dist}(a, K)=\operatorname{dist}(b, K)=\max \left\{\operatorname{dist}_{\widetilde{K}}(a, K), \operatorname{dist}_{\widetilde{K}}(b, K)\right\} .
$$

Therefore, $a \approx_{K} b$.

Assume that $(L \mid K, v)$ is an algebraic extension and $v$ is an extension of the valuation of $L$ to $\widetilde{K}=\widetilde{L}$. Take an element $b$ which is algebraic over $K$ and hence also over $L$. Since $L \mid K$ is algebraic, $\widetilde{v K}=\widetilde{v L}$ and thus $\operatorname{dist}_{\widetilde{L}}(b, L)$ and $\operatorname{dist}_{\widetilde{K}}(b, K)$ are cuts in the same group. Hence we can compare the distances.

Lemma 3.9. Take an algebraic extension $(L \mid K, v)$ and an element b algebraic over $K$. Fix an extension of $v$ to $L(b)$. Then $\operatorname{dist}_{\widetilde{K}}(b, K) \leq \operatorname{dist}_{\tilde{L}}^{(b, L)}$. Moreover, if

$$
\operatorname{dist}_{\widetilde{K}}(b, K)<\operatorname{dist}_{\widetilde{L}}(b, L),
$$

then there is $a \in L$ such that $a \approx_{K} b$. Then in particular,

$$
\operatorname{dist}_{\widetilde{K}}(b, K)=\operatorname{dist}_{\widetilde{K}}(a, K) .
$$

Proof. The first inequality follows immediately from the definition of the distance.

Assume that dist $\widetilde{K}(b, K)<\operatorname{dist}_{\widetilde{L}}(b, L)$. Then there is $a \in L$ such that

$$
v(b-a)>v(b-K) .
$$

Hence for any $c \in K$ we have $v(b-c)=v(b-c-(b-a))=v(a-c)$, which by Lemma 3.5 yields that $a \approx_{K} b$. Thus in particular, $\operatorname{dist}_{\widetilde{K}}(b, K)=\operatorname{dist}_{\widetilde{K}}(a, K)$ by Corollary 3.6.

The above lemma is an analogue of Lemma 2.18 of [17].

Directly from Lemma 2.2 we obtain the following fact. 
Lemma 3.10. Take a defectless extension $(L \mid K, v)$ of henselian fields. Assume that $a_{0}, a_{1} \ldots, a_{n}$ is a standard valuation basis of the extension with $a_{0}=1$. Take an element $b \in L \backslash K$. If $b=\sum_{i=0}^{n} c_{i} a_{i}$, then

$$
\operatorname{dist}_{\tilde{K}}(b, K)=\delta^{+}
$$

with $\delta=v\left(a-c_{0}\right)=\min _{1 \leq i \leq n} v\left(c_{i} a_{i}\right)$.

Take an algebraic extension $(L \mid E, v)$ and assume that $v$ extends in a unique way from $E$ to $L$. Recall that an element $b \in L$ is called weakly immediate over $E$ if the set $v(b-E)$ has no maximal element. By Lemma 2.6, this means that $\operatorname{appr}(b, E)$ is an immediate approximation type. Note that if $b$ is weakly immediate over $E$, then $\infty \notin v(b-E)$, hence the extension $E(b) \mid E$ is nontrivial. Note also that if the extension $L \mid E$ is immediate, then by Theorem 2.1 , every element $b \in L \backslash E$ is weakly immediate over $E$.

Lemma 3.9 together with Lemma 2.2 yields the following fact (cf. also Lemma 7 of [6]).

Corollary 3.11. Take an algebraic extension $(L \mid E, v)$ and a defectless algebraic extension $(F \mid E, v)$ such that $v$ extends in a unique way from $E$ to $F$. Then every $b \in L$ weakly immediate over $E$ is also weakly immediate over $F$, with

$$
\operatorname{dist}_{\widetilde{F}}(b, F)=\operatorname{dist}_{\widetilde{E}}(b, E) .
$$

Another important application of Lemma 3.9 is the case when $L$ is the henselization of $K$. We obtain then the following fact (for the proof see Lemma 9 of [6]).

Proposition 3.12. Take an algebraic extension $L \mid K$ such that the valuation of $K$ admits a unique extension to $L$. Then for every $b \in L \backslash K$,

$$
\operatorname{dist}_{\widetilde{K}}\left(b, K^{h}\right)=\operatorname{dist}_{\widetilde{K}}(b, K) .
$$

Take an extension $(K(a) \mid K, v)$ of degree $n$. We say that the element $a$ is strongly immediate over $K$ if the following conditions hold:

(SI1) the set $v(a-K)$ has no maximal element,

(SI2) for every polynomial $g \in K[X]$ of degree less than $n$ there is $\alpha \in v(a-K)$ such that for every $c \in K$ with $v(a-c) \geq \alpha$ the value $v g(c)$ is fixed.

Note that by Lemma 2.6, condition (SI1) holds if and only if the approximation type appr $(a, K)$ is immediate. Moreover, (SI2) states that the approximation type $\operatorname{appr}(a, K)$ fixes the value of every polynomial of degree less than $n$. Thus if $a$ is strongly immediate over $K$, then part a) of Lemma 2.7, yields that the minimal polynomial of $a$ over $K$ is an associated minimal polynomial for $\operatorname{appr}(a, K)$. Hence, every associated minimal polynomial for $\operatorname{appr}(a, K)$ is of degree $n$. On the other hand, if $g$ is an associated minimal polynomial for $\operatorname{appr}(a, K)$ of degree $n$, then by the definition, appr $(a, K)$ fixes the value of every polynomial of degree less that $n$. We thus obtain the following result. 
Lemma 3.13. Assume that $(K(a) \mid K, v)$ is an extension of degree $n$. Then a is strongly immediate over $K$ if and only if the approximation type appr $(a, K)$ is immediate and every associated minimal polynomial for $\operatorname{appr}(a, K)$ is of degree $n$.

Lemma 3.14. Take an algebraic extension $(L \mid K, v)$ and an element $a \in L$ strongly immediate over $K$. If $b \in L$ is such that $[K(a): K] \geq[K(b): K]$ and $a \approx_{K} b$, then $[K(a): K]=[K(b): K]$ and $b$ is strongly immediate over $K$.

Proof. Since $a \approx_{K} b$, Lemma 3.4 we obtain that $\operatorname{appr}(a, K)=\operatorname{appr}(b, K)$. As $a$ is strongly immediate over $K$, the approximation type appr $(a, K)=\operatorname{appr}(b, K)$ fixes the value of every polynomial of degree less than $[K(a): K]$. By Lemma 2.7, the approximation type $\operatorname{appr}(b, K)$ does not fix the value of a minimal polynomial of $b$ over $K$. Hence, $[K(a): K] \leq[K(b): K]$ and thus $[K(a): K]=[K(b): K]$.

Since $a$ is strongly immediate, $v(a-K)$ has no maximal element. Furthermore, relation $a \approx_{K} b$ together with Lemma 3.5 yields that $v(b-K)=v(a-K)$. Hence $v(b-K)$ has no maximal element. Consequently, $b$ is strongly immediate over $K$.

If $(K(a) \mid K, v)$ is an immediate extension, then by Theorem 2.1 the set $v(a-K)$ has no maximal element. Lemma 2.21 of [17] shows that if $p=\operatorname{char} K v>0$, the converse holds for extensions of degree $p$. The next lemma shows that it holds also for extensions generated by strongly immediate elements.

Lemma 3.15. Take an algebraic extension $(K(a) \mid K, v)$ and assume that $a$ is strongly immediate over $K$. Then the following assertions hold.

1) The extension $(K(a) \mid K, v)$ is immediate.

2) If $b \in \widetilde{K}$ is such that $a \approx_{K} b$ and $[K(a): K] \geq[K(b): K]$, then the extension $(K(b) \mid K, v)$ is immediate.

Proof. Since $a$ is strongly immediate, by Lemma 3.13 the approximation type appr $(a, K)$ is immediate. Lemma 2.7 yields that appr $(a, K)$ is an algebraic approximation type. Take any element $b \in K(a)$. Then $b=h(a)$ for some polynomial $h \in K[X]$ of degree less than $[K(a): K]$. From the previous lemma and part b) of Lemma 2.7 it follows that $\operatorname{appr}(b, K)=\operatorname{appr}(h(a), K)$ is an immediate approximation type. Hence by Lemma 2.6, the extension $(K(a) \mid K, v)$ is immediate.

Part 2) follows directly from part 1) and Lemma 3.14.

Part 1) of Lemma 3.15 together with Theorem 2.1 yield the following property.

Corollary 3.16. Take an algebraic extension $(K(a) \mid K, v)$ such that a has property (SI2). The extension is immediate if and only if the set $v(a-K)$ has no maximal element.

The next example shows that the above equivalence is not true in general. 
Example 3.17. Consider the power series field $\mathbb{F}_{p}\left(\left(x^{\mathbb{Q}}\right)\right)$ with the canonical valuation $v$. We denote the restriction of the canonical valuation of $\mathbb{F}_{p}\left(\left(x^{\mathbb{Q}}\right)\right)$ to any subfield of $\mathbb{F}_{p}\left(\left(x^{\mathbb{Q}}\right)\right)$ again by $v$. Set

$$
K:=\mathbb{F}_{p}\left(x^{1 / p^{i}} \mid i \in \mathbb{N}\right) \subseteq \mathbb{F}_{p}\left(\left(x^{\mathbb{Q}}\right)\right),
$$

which is the perfect hull of the rational function field $\mathbb{F}_{p}(x)$. Then $v K$ is the $p$-divisible hull $\frac{1}{p^{\infty}} \mathbb{Z}$ of $\mathbb{Z}$ and $K v=\mathbb{F}_{p}$. Set

$$
a:=\sum_{i=1}^{\infty} x^{-1 / p^{i}} \in \mathbb{F}_{p}\left(\left(x^{\mathbb{Q}}\right)\right) .
$$

Note that $a^{p}-a=\frac{1}{x}$. Since $a^{p^{n}} \notin \mathbb{F}_{p}(x)$ for every $n \in \mathbb{N}$, the element $a$ does not lie in $K$. In Example 3.12 of [19] it is shown that $v(a-K)=\frac{1}{p^{\infty}} \mathbb{Z}^{<0}$, where $\frac{1}{p^{\infty}} \mathbb{Z}^{<0}$ is the set of all negative elements of $\frac{1}{p^{\infty}} \mathbb{Z}$. Take any prime $q \neq p$ and consider the element $a+x^{1 / q} \in \mathbb{F}_{p}\left(\left(x^{\mathbb{Q}}\right)\right)$. Since $v x^{1 / q}=\frac{1}{q}>v(a-K)$ we obtain that

$$
v\left(a+x^{1 / q}-K\right)=v(a-K)=\frac{1}{p^{\infty}} \mathbb{Z}^{<0} .
$$

In particular, the set $v\left(a+x^{1 / q}-K\right)$ has no maximal element.

On the other hand, the extension $\left(K\left(a+x^{1 / q}\right) \mid K, v\right)$ is not immediate. Indeed, for an element $\left(a+x^{1 / q}\right)^{p}-\left(a+x^{1 / q}\right)-\frac{1}{x} \in K\left(a+x^{1 / q}\right)$ we have

$v\left(\left(a+x^{1 / q}\right)^{p}-\left(a+x^{1 / q}\right)-\frac{1}{x}\right)=v\left(x^{p / q}-x^{1 / q}\right)=\frac{1}{q} \notin \frac{1}{p^{\infty}} \mathbb{Z}=v K$.

Proposition 3.18. Take an algebraic extension $(K(a) \mid K, v)$ such that a has property (SI2) and fix an extension of $v$ to $\widetilde{K}$. If $b \in \widetilde{K}$ is such that $a \approx_{K} b$, then for every polynomial $f \in K[X]$ of degree less than $[K(a): K]$,

$$
f(a) \approx_{K} f(b) .
$$

For the proof we will need the following property (cf. Lemma 5.2 of [23]).

Lemma 3.19. Take an algebraic approximation type $\operatorname{appr}(a, K)$ and a polynomial $g \in K[X]$ of degree less than or equal to the degree of an associated minimal polynomial for $\operatorname{appr}(a, K)$. Assume that $\operatorname{appr}(a, K)$ fixes the value of $g$ and take an element $\alpha \in v(a-K) \cap v K_{\infty}$ such that $v g(c)$ is fixed for every $c \in K$ with $v(a-c) \geq \alpha$. Then $v g(a)=v g(c)$.

Proof of Lemma 3.18. As $a \approx_{K} b$, by Lemma 3.4 we obtain that $\operatorname{appr}(a, K)=$ $\operatorname{appr}(b, K)$. By the assumption on $K(a) \mid K$, the approximation type $\operatorname{appr}(a, K)$ fixes the value of every polynomial of degree less than $n:=[K(a): K]$. Take such a polynomial $f$ and an element $c \in K$. Then also $\operatorname{deg}(f-c)<n$. Hence there is $\alpha \in v(a-K)$ such that for all $d \in K$ with $v(a-d) \geq \alpha$ the value $v((f-c)(d))=v(f(d)-c)$ is fixed. Lemma 3.5 yields that $\alpha \in v(b-K)$ and 
for every $d \in K$ with $v(b-d) \geq \alpha$ the value $v(f(d)-c)$ is fixed. Applying the above lemma to $g=f-c$ and elements $a$ and $b$ respectively, we obtain that

$$
v(f(a)-c)=v(f(d)-c)=v(f(b)-c) .
$$

Since the equality $v(f(a)-c)=v(f(b)-c)$ holds for every $c \in K$, by Lemma 3.5 we obtain the relation $f(a) \approx_{K} f(b)$.

We show now how the distances of any elements in an immediate simple extension generated by a strongly immediate element depend on the distance of the generator of the extensions. In the remaining part of the section assume that the residue field $E v$ of $(E, v)$ is of positive characteristic $p$.

Lemma 3.20. Assume that $(E(a) \mid E, v)$ is an extension of degree $p^{j}$ such that the valuation $v$ admits a unique extension from $E$ to $E(a)$. If a is strongly immediate over $K$, then for every nonconstant polynomial $f \in E[X]$ of degree less than $[E(a): E]$ there is $\alpha \in v E$ and $i \in\{0, \ldots, j-1\}$ such that

$$
\operatorname{dist}_{\widetilde{E}}(f(a), E)=\alpha+p^{i} \operatorname{dist}_{\widetilde{E}}(a, E) .
$$

Proof. Since $a$ is strongly immediate, property (SI1) together with part 1) of Lemma 3.1 yield that $\operatorname{dist}_{\widetilde{E}}(a, E)=\operatorname{dist}(a, E)$ and $\operatorname{dist}_{\widetilde{E}}(f(a), E)=$ dist $(f(a), E)$. Moreover, Lemma 3.13 implies that $\operatorname{appr}(a, E)$ is an immediate approximation type. Now the assertion of the lemma follows from Proposition 7.4 and Lemma 8.2 of [23].

Corollary 3.21. If $E(a) \mid E$ is an immediate extension of degree $p$ such that the valuation $v$ admits a unique extension from $E$ to $E(a)$, then for every $b \in E(a) \backslash E$, there is $\alpha \in v E$ such that

$$
\operatorname{dist}_{\widetilde{E}}(b, E)=\alpha+\operatorname{dist}_{\widetilde{E}}(a, E) .
$$

Proof. By Theorem 2.1 the set $v(a-E)$ has no maximal element. Together with Lemma 11 of [6] this yields that the element $a$ is strongly immediate over $K$. Now the assertion follows from the previous lemma.

\section{Distances of elements in extensions of prime degree}

We apply now the notions of the distance and the relation $\approx_{K}$ considered in the previous section to the case of extensions $(K(a) \mid K, v),(K(b) \mid K, v)$ of prime degree. We show that for such extensions the relation $a \approx_{K} b$ implies a strong connection between the value group extensions and between the residue field extensions of $(K(a) \mid K, v)$ and $(K(b) \mid K, v)$. We also consider possible distances of elements in extensions of prime degree.

Throughout this section we assume that $(K, v)$ is a valued field, fix an extension of $v$ to the algebraic closure $\widetilde{K}$ of $K$ and denote it again by $v$, unless stated otherwise. Denote by $K^{h}$ the henselization of $K$ with respect to the fixed extension of $v$. Furthermore, we take $p$ to be a prime number. 
Take an extension $K(a) \mid K$ of degree $p$ such that the valuation $v$ admits a unique extension from $K$ to $K(a)$. Then from the Lemma of Ostrowski it follows that either $(K(a) \mid K, v)$ is an immediate extension (which is possible only in the case of $p=$ char $K v),(v K(a): v K)=p$ and the residue field extension is trivial, or $[K(a) v: K v]=p$ and the value group extension is trivial.

Assume that $(v K(a): v K)=p$ or $[K(a) v: K v]=p$. Then the extension $K(a) \mid K$ is defectless. Since $v$ extends in a unique way from $K$ to $K(a)$, the extensions $K(a)$ and $K^{h}$ are linearly disjoined over $K$. This together with Eq. (2) yields that $K(a)^{h}=K^{h}(a)$ is a defectless extension of $K^{h}$ degree $p$. Moreover, as $K^{h}(a)=K(a)^{h}$ and the henselization is an immediate extension of a valued field, we obtain that $\left(v K^{h}(a): v K^{h}\right)=(v K(a): v K)=p$ or $\left[K^{h}(a) v: K^{h} v\right]=[K(a) v: K v]=p$. Hence Lemma 2.2 yields that $v\left(a-K^{h}\right)$ admits a maximal element. As dist $\widetilde{K}(a, K)=\operatorname{dist}_{\widetilde{K}}\left(a, K^{h}\right)$ by Proposition 3.12, we deduce that $v(a-K)$ has a maximal element, equal to the maximal element of $v\left(a-K^{h}\right)$.

If the value group extension $v K(a) \mid v K$ is nontrivial, then $\left[K(a)^{h} v: K^{h} v\right]=1$ and by Lemma 3.1 the maximal element $\alpha$ of $v\left(a-K^{h}\right)$, hence also of $v(a-K)$, lies in $v K^{h}(a) \backslash v K^{h}=v K(a) \backslash v K$. Thus $\alpha$ has order $p$ modulo $v K$ and $v K(a)=$ $v K+\alpha \mathbb{Z}$. Obviously, if $v(a-K)$ has a maximal element $\alpha \notin v K$, then the value group extension is nontrivial and as before we deduce that $v K(a)=v K+\alpha \mathbb{Z}$.

A similar argument together with Lemma 3.1 shows that the residue field extension $K(a) v \mid K v$ is nontrivial if and only if $v(a-K)$ admits a maximal element which lies in $v K$. Then $K(a) v=K v\left(d^{-1}(a-c) v\right)$ for $c, d \in K$ such that $v(a-c)=\alpha=v d$.

By Theorem 2.1 we obtain that if $K(a) \mid K$ is an immediate extension, then the set $v(a-K)$ has no maximal element. Note that also the converse holds. It follows from the fact that if the extension $K(a) \mid K$ is not immediate and $v$ extends in a unique way from $K$ to $K(a)$, then the above arguments show that $K(a) \mid K$ is defectless and $v(a-K)$ admits a maximal element.

We thus can read off the information about the value group and the residue field extensions from the distance.

Lemma 4.1. Take an extension $K(a) \mid K$ of degree $p$ and assume that the valuation $v$ of $K$ extends in a unique way to $K(a)$.

1) The set $v(a-K)$ has no maximal element if and only if the extension $(K(a) \mid K, v)$ is immediate.

2) The set $v(a-K)$ admits a maximal element $\alpha \notin v K$ if and only if the value group extension $v K(a) \mid v K$ is nontrivial. If this holds, then $v K(a)=v K+\alpha \mathbb{Z}$.

3) The set $v(a-K)$ admits a maximal element $\alpha \in v K$ if and only if the residue field extension $K(a) v \mid K v$ is nontrivial. If this holds, then for every $c, d \in K$ such that $v(a-c)=\alpha=v d$ we have $K(a) v=K v\left(d^{-1}(a-c) v\right)$.

Proposition 4.2. Take extensions $K(a) \mid K$ and $K(b) \mid K$ of degree $p$ and assume that the valuation $v$ of $K$ extends in a unique way to the fields $K(a)$ and $K(b)$. Assume that $a \approx_{K} b$. Then

$$
v K(a)=v K(b) \text { and }[K(a) v: K v]=[K(b) v: K v] .
$$


If moreover $v(b-a)>\operatorname{dist}_{\widetilde{K}}(a, K)$, then $K(a) v=K(b) v$.

Proof. Note that since $a \approx_{K} b$, Lemma 3.5 yields that $v(a-K)=v(b-K)$.

Consider the extension $(K(a) \mid K, v)$. As we have already seen, we have three possible cases: the extension is immediate, $(v K(a): v K)=p$ and the residue field extension is trivial, or $[K(a) v: K v]=p$ and the value group extension is trivial. The same holds for $(K(b) \mid K, v)$.

Suppose that $K(a) \mid K$ is immediate. Then $v(a-K)$ has no maximal element and thus also the set $v(b-K)$ has no maximal element. Now the assertion of the proposition follows from Lemma 2.21 of [17] .

Assume that the group $v K(a) / v K$ is nontrivial. Then by the previous lemma, $v(b-K)=v(a-K)$ has a maximal element $\alpha \notin v K$. Together with Lemma 4.1 this yields that $v K(b)=v K+\alpha \mathbb{Z}=v K(a)$. Moreover, both extensions $K(a) v \mid K v$ and $K(b) v \mid K v$ are trivial.

Suppose now that the extension $K(a) v \mid K v$ is nontrivial. Then by the previous lemma, $v(b-K)=v(a-K)$ admits a maximal element $\alpha \in v K$. Hence, Lemma 4.1 yields that the extension $K(b) v \mid K v$ is nontrivial and $v K(a)=v K=$ $v K(b)$.

Assume additionally that $v(a-b)>\operatorname{dist}_{\widetilde{K}}(a, K)$. If $c, d \in K$ are such that $v(a-c)=\alpha=v d$, then by Lemma 4.1 the element $\xi:=d^{-1}(a-c) v$ generates the extension $K(a) v \mid K v$. By the assumption on dist $\widetilde{K}^{(a, K)}=\operatorname{dist}_{\widetilde{K}}(b, K)$ we obtain inequality $v(b-a)>\alpha=v d$. Hence

$$
v\left(d^{-1}(b-c)-d^{-1}(a-c)\right)=v\left(d^{-1}(a-b)\right)>0 .
$$

Thus $d^{-1}(b-c) v=d^{-1}(a-c) v$ and $\xi \in K(b) v$. From the fact that $K(b) v \mid K v$ is of prime degree, we deduce that $K(b) v=K v(\xi)=K(a) v$.

The next example shows that the additional condition $v(b-a)>\operatorname{dist}_{\widetilde{K}}(a, K)$ is necessary to obtain the last assertion of the above proposition.

Example 4.3. Assume that $(K, v)$ is henselian and the residue field $K v$ admits distinct separable extensions $K v(\eta) \mid K v$ and $K v(\vartheta) \mid K v$ of degree $p$. Choose monic polynomials $f, g \in \mathcal{O}_{K}[X]$ of degree $p$ whose reductions modulo $v$ are the minimal polynomials of $\eta$ and $\vartheta$, respectively. Then $1 f$ admits a root $a \in \widetilde{K}$ such that $a v=\eta$ and $g$ admits a root $b \in \widetilde{K}$ such that $a v=\vartheta$. This yields that $[K(a): K]=p=$ $[K v(\eta): K v]$ and $\left(1, a, \ldots, a^{p-1}\right)$ is a standard valuation basis of $(K(a) \mid K, v)$. Similarly, $[K(b): K]=p=[K v(\vartheta): K v]$ and $\left(1, b, \ldots, b^{p-1}\right)$ is a standard valuation basis of $(K(b) \mid K, v)$.

Lemma 3.10 yields that dist $\widetilde{K}_{(}(a, K)=(v a)^{+}=0^{+}$. Similarly, dist $\widetilde{K}(b, K)=$ $(v b)^{+}=0^{+}$. On the other hand, $v(a-b)=0$, since otherwise $v(a-b)>0$, which would mean that $\eta=a v=b v=\vartheta$, a contradiction. We thus obtain that

$$
v(a-b)=0=\max \left\{\operatorname{dist}_{\widetilde{K}}(a, K), \operatorname{dist}_{\widetilde{K}}(b, K)\right\}
$$

and hence $a \approx_{K} b$, but $K(a) v=K v(\eta) \neq K v(\vartheta)=K(b) v$.

Take an extension $L \mid K$ of degree $p$. We consider which distances dist $\widetilde{K}_{K}(a, K)$ are possible for elements $a \in L \backslash K$. Lemma 3.9 yields that $\operatorname{dist}_{\widetilde{K}}(a, K)=$ 
$\operatorname{dist}_{\widetilde{K}}\left(a, K^{h}\right)$ or there is $b \in K^{h}$ such that $\operatorname{dist}_{\widetilde{K}}(a, K)=\operatorname{dist}_{\widetilde{K}}(b, K)$. The next theorem determines dist $\widetilde{K}(a, K)$ in the second case (for the proof see [18], Theorem 1).

Theorem 4.4. Every element $d \in K^{h} \backslash K$ satisfies the equality

$$
\operatorname{dist}_{\widetilde{K}}(d, K)=(\alpha+H)^{+}
$$

for some element $\alpha \in v K$ and a nontrivial convex subgroup $H$ of $v K$.

In particular, if the valuation $v$ of $K$ is of rank 1 , then $\operatorname{dist}_{\widetilde{K}}(d, K)=\infty$.

Theorem 4.4 generalizes the well-known fact that if $v$ is a rank 1 valuation of a field $K$, then the henselization of $(K, v)$ is contained in the completion of the field.

Proposition 3.12 shows that the case $\operatorname{dist}_{\widetilde{K}}(a, K)=\operatorname{dist}_{\widetilde{K}}\left(a, K^{h}\right)$ holds in particular if $v$ admits a unique extension from $K$ to $K(a)$, that is, if $K(a)$ is linearly disjoint from $K^{h}$ over $K$. The next proposition describes the possible distances if such an extension is defectless.

Proposition 4.5. Take a defectless extension $(L \mid K, v)$ of degree $p$ and assume that the valuation $v$ of $K$ extends in a unique way to $L$.

1) If $v L=v K$, then the distance of every element $b \in L \backslash K$ from $K$ over $\widetilde{K}$ is of the form $\alpha^{+}$for some $\alpha \in v K$. Conversely, for every $\alpha \in v K$ there is $b \in L \backslash K$ such that dist $\widetilde{K}(b, K)=\alpha^{+}$.

2) If the value group extension $v L \mid v K$ is nontrivial, then the distance of every element $b \in L \backslash K$ from $K$ over $\widetilde{K}$ is of the form $\alpha^{+}$for some $\alpha \in v L \backslash v K$. Furthermore, for every $\alpha \in v L \backslash v K$ there is $b \in L \backslash K$ such that dist $\widetilde{K}(b, K)=\alpha^{+}$.

Proof. Note first, that by Proposition 3.12, for every $b \in L \backslash K$ we have $\operatorname{dist}_{\widetilde{K}}(b, K)=\operatorname{dist}_{\widetilde{K}}\left(b, K^{h}\right)$. Since $v$ extends in a unique way from $K$ to $L$, the extensions $L \mid K$ and $K^{h} \mid K$ are linearly disjoint. Thus $L . K^{h} \mid K^{h}$ is an extension of degree $p$. Moreover, $v K^{h}=v K, K^{h} v=K v$ and $v L . K^{h}=v L^{h}=v L$, $L . K^{h} v=L^{h} v=L v$. Hence, we may assume that $(K, v)$ is henselian.

Assume first that $v L=v K$. As $(L \mid K, v)$ is defectless, the fundamental equality yields that $[L v: K v]=p$. Take any $a \in L \backslash K$. Since $L \mid K$ is of prime degree, $L=K(a)$ and Lemma 4.1 yields that dist $\widetilde{K}(a, K)=\alpha^{+}$for some $\alpha \in v K$.

Fix an element $\beta \in v K$. Take any $a \in L \backslash K$. As we have seen, $\operatorname{dist}_{\widetilde{K}}(a, K)=$ $\alpha^{+}$for some $\alpha \in v K$. Take $c \in v K$ such that $v c=\beta-\alpha$. Then by Lemma 3.2 we obtain that dist $\widetilde{K}(c a, K)=\beta^{+}$.

Assume that $v L \mid v K$ is nontrivial. Then $v L=v K+\gamma \mathbb{Z}$ for some element $\gamma \in \widetilde{v K}$ of order $p$ modulo $v K$. Similarly to the previous case, we deduce that for an element $a \in L$ Lemma 4.1 yields that dist $\widetilde{K}(a, K)=\alpha^{+}$for some $\alpha \in v L \backslash v K$.

On the other hand, if $\beta$ is an element of $v L \backslash v K$, then it is of the form $v c+i \gamma$ for some $c \in K$ and $1 \leq i \leq p-1$. Take an element $a \in L$ such that $v a=\gamma$. Then for $b=c a^{i}$ Lemma 3.10 yields that

$$
\operatorname{dist}_{\widetilde{K}}(b, K)=\left(v\left(c a^{i}\right)\right)^{+}=\beta^{+} .
$$


Assume now that $(L \mid K, v)$ is an immediate extension such that $v$ extends in a unique way from $K$ to $L$. Recall that then dist $\widetilde{K}(b, K)=\operatorname{dist}(b, K)$ for any $b \in L \backslash K$. Moreover, the Lemma of Ostrowski yields that $p=$ char $K v$ and $L \mid K$ is a defect extension. Since $L^{h}=K^{h} . L$, Eq. (2) yields that $d\left(L . K^{h} \mid K^{h}, v\right)=d(L \mid K, v)=$ $p$. Hence $\left(L . K^{h} \mid K^{h}, v\right)$ is a defect extension of degree $p$. Furthermore, by Proposition 3.12 for any element $b \in L \backslash K$ we have dist $\widetilde{K}(b, K)=\operatorname{dist} \widetilde{K}\left(b, K^{h}\right)$. This shows that we can assume additionally that $(K, v)$ is henselian.

Take a defect extension $(L \mid K, v)$ of degree $p=\operatorname{char} K v$. Then $v$ extends in a unique way from $K$ to $L$. Hence Proposition 3.12 shows that for every $b \in L \backslash K$

$$
\operatorname{dist}_{\widetilde{K}}(b, K)=\operatorname{dist}_{\widetilde{K}}\left(b, K^{h}\right) .
$$

We investigate now the form of distances of elements in defect extensions of degree $p$ in the case of henselian valued fields of equal positive characteristic $p$.

Proposition 4.6. Assume that $(K, v)$ is henselian, of positive characteristic $p$ and $(L \mid K, v)$ is a separable defect extension of degree $p$. If $b \in L \backslash K$, then one of the following cases holds:

1) dist $\widetilde{K}(b, K)=\alpha+H^{-}$for some $\alpha \in \frac{1}{p-1} v K$ and a proper convex subgroup $H$ of $v K$;

2) $\operatorname{dist}_{\widetilde{K}}(b, K)=\operatorname{dist}_{\widetilde{E}}(d, E)$ for some cyclic tame extension $(E \mid K, v)$ of degree dividing $p-1$ (possibly trivial) and $d \in E^{1 / p}$ such that the extension $(E(d) \mid E, v)$ is immediate.

Proof. Since $b \in L \backslash K$ and $L \mid K$ is of prime degree, we have $L=K(b)$. Moreover, since $L \mid K$ is a defect extension of prime degree, it is immediate.

Note that by Lemma 2.5 there is a tame cyclic extension $E \mid K$ of degree dividing $p-1$ such that $(L . E \mid E, v)$ is an Artin-Schreier defect extension. Since the extension $E \mid K$ is tame, it is in particular defectless. Thus by Corollary 3.11, we obtain that dist $\widetilde{K}(b, K)=\operatorname{dist}_{\widetilde{E}}(b, E)$. Take $\vartheta$ to be an Artin-Schreier generator of $L . E \mid E$. Then $L . E=E(\vartheta)$. Since also $L . E=E(b)$, we obtain that $b$ is an element of $E(\vartheta)$ which does not lie in $E$. Corollary 3.21 shows that

$$
\operatorname{dist}_{\widetilde{K}}(b, K)=\operatorname{dist}_{\widetilde{E}}(b, E)=\alpha+\operatorname{dist}_{\widetilde{E}}(\vartheta, E)
$$

for some $\alpha \in v E$. As $[E: K]$ divides $p-1$, also $(v E: v K)$ divides $p-1$. Hence, $v E \subseteq \frac{1}{p-1} v K$.

Assume first that $E(\vartheta) \mid E$ is an independent Artin-Schreier defect extension. Then Proposition 2.4 shows that dist $\widetilde{E}(\vartheta, E)=H^{-}$for some proper convex subgroup of $\widetilde{v E}=\widetilde{v K}$. Hence by Eq. (6) we obtain that

$$
\operatorname{dist}_{\widetilde{K}}(b, K)=\alpha+H^{-}
$$

for some proper convex subgroup $H$ of $\widetilde{v K}$ and an element $\alpha \in \frac{1}{p-1} v K$.

If $L \mid K$ is a dependent Artin-Schreier defect extension, then there is $a \in E$ such that $a \approx_{E} \vartheta$ and thus $\operatorname{dist}_{\widetilde{E}}(\vartheta, E)=\operatorname{dist}_{\widetilde{E}}\left(a^{1 / p}, E\right)$. Hence if $c \in E$ is such that $v c=\alpha$, by Lemma 3.2 and Eq. (6)

$$
\operatorname{dist}_{\widetilde{K}}(b, K)=v c+\operatorname{dist}_{\widetilde{E}}\left(a^{1 / p}, E\right)=\operatorname{dist}_{\widetilde{E}}\left(c a^{1 / p}, E\right) .
$$

Setting $d=c a^{1 / p}$ we obtain case 2 ) of the proposition. 
Note that if $L \mid K$ is itself an Artin-Schreier defect extension, then we can take $E=K$ and replace $\alpha \in \frac{1}{p-1} v K$ in case 1 ) of the above proposition by $\alpha \in v K$.

The distances in case 1) of the above proposition are determined by the convex subgroups of $\widetilde{v K}$. Thus we are left with the distances of those elements which generate purely inseparable immediate extensions of degree $p$.

Note that by Theorem 2.1, if $(K(b) \mid K, v)$ is an immediate purely inseparable extension, then the set $v(b-K)$ admits no maximal element. Because of this, $\operatorname{dist}_{\widetilde{K}}(b, K)$ may not be anymore a cut induced by some element of $v K(b)$, like in the case of defectless extensions.

In the following facts we consider cuts in the ordered abelian group $\mathbb{Q}$. For simplicity we generalize our notation by setting

$$
\delta^{-}=(\{\alpha \in \mathbb{Q} \mid \alpha<\delta\},\{\alpha \in \mathbb{Q} \mid \alpha \geq \delta\}) .
$$

for any element $\delta \in \mathbb{R}$. We also identify the distances $\infty^{-}$and $\infty$.

Theorem 4.7. Take a field $K$ of positive characteristic $p$, a non-discrete subgroup $\Gamma$ of $\mathbb{Q}$ which is not $p$-divisible and $\delta \in \mathbb{R} \cup\{\infty\}$. Then there is a valuation $v$ on the rational function field $K(x, y) \mid K$ whose restriction to $K$ is trivial, such that $v K(x, y)=\Gamma$, the extension $\left(K\left(x, y^{1 / p}\right) \mid K(x, y), v\right)$ is immediate and $\operatorname{dist}\left(y^{1 / p}, K(x, y)\right)=\delta^{-}$.

Note that if the extension $K\left(x, y^{1 / p}\right) \mid K(x, y)$ is immediate, then

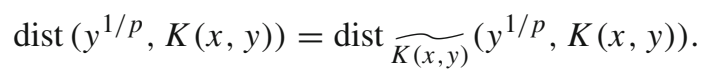

In the proof of the above theorem we give a construction of a valuation of the function field $K(x, y)$ which satisfies the assertion of the above theorem. We first prove lemmas which we use in the construction of our valued field extension.

Lemma 4.8. Take a field $K$ of positive characteristic $p$ and the power series field $K\left(\left(x^{\mathbb{Q}}\right)\right)$. For a prime number $q \neq p$ take

$$
y=\sum_{i=1}^{\infty} x^{p \gamma_{i} q^{-e_{i}}} \in K\left(\left(x^{\mathbb{Q}}\right)\right),
$$

where $\gamma_{i}=\frac{n_{i}}{r_{i}}$ and $r_{i}, n_{i} \in \mathbb{Z}$ are coprime and not divisible by $q$. Assume moreover that $\left(e_{i}\right)_{i \in \mathbb{N}}$ is a sequence of natural numbers such that $e_{i+1}-e_{i} \geq i$, for all $i \in \mathbb{N}$. Suppose that the sequence $\left(\gamma_{i} q^{-e_{i}}\right)_{i \in \mathbb{N}} i$ strictly increasing and convergent to $\delta \in \mathbb{R}$. If $K\left(x, y^{1 / p}\right)$ is equipped with the restriction $v$ of the canonical valuation of $K\left(\left(x^{\mathbb{Q}}\right)\right)$, then

$$
\operatorname{dist} \underset{K(x, y)}{\widetilde{1 / p}}, K(x, y)) \leq \delta^{-} .
$$

Proof. Note first that it is enough to show that $v\left(y^{1 / p}-f\right)<\delta$ for every element $f \in K\left[x, \frac{1}{x}, y\right]$. Indeed, since by Lemma $2.8 K\left[x, \frac{1}{x}, y\right]$ is dense in $K(x, y)$, for every $u \in K(x, y)$ there is $f \in K\left[x, \frac{1}{x}, y\right]$ such that $v(f-u)>\delta$. Then

$$
v\left(y^{1 / p}-u\right)=v\left(y^{1 / p}-f+f-u\right)=v\left(y^{1 / p}-f\right)<\delta .
$$


Take an element $f \in K\left[x, \frac{1}{x}, y\right]$. Then

$$
f=\sum_{\substack{-n \leq i \leq m \\ 0 \leq j \leq l}} a_{i j} x^{i} y^{j}
$$

for some $a_{i j} \in K$ and $n, m, l \in \mathbb{N} \cup\{0\}$. For every $i \in\{-n, \ldots, m\}$ and $j \in$ $\{0, \ldots, l\}$ set

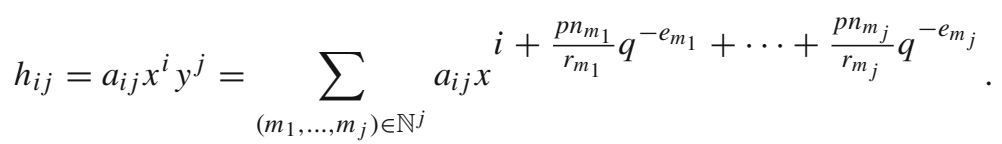

We show that for each $j$ there is $N_{j} \in \mathbb{N}$ such that for every $i \in\{-n, \ldots, m\}$ and $N \geq N_{j}$ we have $\frac{n_{N}}{r_{N}} q^{-e_{N}} \notin \operatorname{supp} h_{i j}$. Since $y^{1 / p}=\sum_{i=1}^{\infty} x^{\frac{n_{i}}{r_{i}} q^{-e_{i}}}$, this means that supp $h_{i j}$ and supp $y^{1 / p}$ have at most finitely many common elements. Note that for $j=0$ we can choose $N_{j}=1$. Take now $j \in\{1, \ldots, l\}$ and suppose that

$$
\frac{n_{N}}{r_{N}} q^{-e_{N}}=i+\frac{p n_{m_{1}}}{r_{m_{1}}} q^{-e_{m_{1}}}+\cdots+\frac{p n_{m_{j}}}{r_{m_{j}}} q^{-e_{m_{j}}}
$$

for some $N, m_{1}, \ldots, m_{j} \in \mathbb{N}$ and $i \in\{-n, \ldots, m\}$. Without loss of generality we can assume that $m_{1} \leq \ldots \leq m_{j}$ and exactly the $k$ last indices are equal, that is $m_{j-k}<m_{j-k+1}=\ldots=m_{j}$. Then $m_{j} \geq N$, as $n_{t}$ and $r_{t}$ are coprime with $q$. Take $r=\operatorname{lcm}\left(r_{m_{1}}, \ldots, r_{m_{j}}, r_{N}\right)$ and set $n_{N}^{\prime}=\frac{r}{r_{N}} n_{N}, n_{m_{t}}^{\prime}=\frac{r}{r_{m_{t}}} n_{m_{t}}$ for $t \leq j$. Since $n_{t}$ and $r_{t}$ are coprime with $q$, also $n_{N}^{\prime}$ and $n_{m_{t}}^{\prime}$ are coprime with $q$. Multiplying both sides of Eq. (8) by $r q^{e_{m_{j}}}$ we obtain

$$
n_{N}^{\prime} q^{e_{m_{j}}-e_{N}}=r q^{e_{m_{j}}} \cdot i+p n_{m_{1}}^{\prime} q^{e m_{j}-e_{m_{1}}}+\cdots+p n_{m_{j-k}}^{\prime} q^{e_{m_{j}}-e_{m_{j-k}}}+k p n_{m_{j}}^{\prime} .
$$

Suppose first that $m_{j}>N$. Then by the assumptions on $e_{t}$ we obtain that $e_{m_{j}}-e_{N} \geq e_{N+1}-e_{N} \geq N$ and $e_{m_{j}}-e_{m_{t}} \geq e_{m_{j}}-e_{m_{j-1}} \geq m_{j}-1 \geq N$ for all $t \leq j-k$. Hence, Eq. (9) yields that $q^{N}$ divides $k p n_{m_{j}}^{\prime}$. Since $n_{m_{j}}^{\prime}$ and $p$ are coprime with $q$ we obtain that $q^{N}$ divides $k$.

Assume now that $m_{j}=N$. Then by (9) we obtain that

$$
n_{N}^{\prime}(k p-1)=-\left(r q^{e_{N}} \cdot i+p n_{m_{1}}^{\prime} q^{e_{N}-e_{m_{1}}}+\cdots+p n_{m_{j-k}}^{\prime} q^{e_{N}-e_{m_{j}-k}}\right) .
$$

Note that since $p$ is a prime and $n_{N}^{\prime}, k$ are natural numbers, the left hand side of the above equation is nonzero. Therefore $i \neq 0$ or $j \neq k$. As in the previous case we show that $e_{N}-e_{m_{t}} \geq N-1$ for $t \leq j-k$. Hence, the right hand side of Eq. (10) is divisible by $q^{N-1}$. Since $n_{N}^{\prime}$ is coprime with $q$, it follows that $q^{N-1}$ divides $k p-1$.

Take $N_{j} \in \mathbb{N}$ such that $q^{N_{j}-1}>p j-1$ and a natural number $N \geq N_{j}$. Since $k \leq j$, we obtain that $q^{N-1}>k p-1$ and consequently $q^{N}>k$. Therefore $q^{N-1}$ does does not divide $k p-1$ and $q^{N}$ does not divide $k$. This yields that Eq. (8) does not hold for any $i, m_{1}, \ldots, m_{j} \in \mathbb{N}$. Hence $\frac{n_{N}}{r_{N}} e^{-e_{N}} \notin \operatorname{supp} h_{i j}$ for $N \geq N_{j}$ and $i \in\{-n, \ldots, m\}$. 
Set $N_{f}=\max \left\{N_{j} \mid 0 \leq j \leq l\right\}$. Then $\frac{n_{N_{f}}}{r_{N_{f}}} q^{-e_{N_{f}}} \notin \operatorname{supp} h_{i j}$ for all $i \in\{-n, \ldots, m\}$ and $j \in\{0, \ldots, l\}$. Consequently,

$$
v\left(y^{1 / p}-f\right) \leq \frac{n_{N_{f}}}{r_{N_{f}}} q^{-e_{N_{f}}}<\delta .
$$

Lemma 4.9. Take a field $K$ of positive characteristic $p$ and the power series field $K\left(\left(x^{\mathbb{Q}}\right)\right)$. Take

$$
y=\sum_{i=1}^{\infty} x^{\frac{p n_{i}}{s_{i}}} \in K\left(\left(x^{\mathbb{Q}}\right)\right)
$$

where $s_{i} \geq 2, i \in \mathbb{N}$ form a strictly increasing sequence of pairwise coprime natural numbers and $\left(n_{i}\right)_{i \in \mathbb{N}}$ is a sequence of integers such that $\operatorname{gcd}\left(s_{i}, n_{i}\right)=1$ for each $i$. Suppose that the sequence $\left(\frac{n_{i}}{s_{i}}\right)_{i \in \mathbb{N}}$ is strictly increasing and convergent to $\delta \in \mathbb{R}$. If $K\left(x, y^{1 / p}\right)$ is equipped with the restriction $v$ of the canonical valuation of $K\left(\left(x^{\mathbb{Q}}\right)\right)$, then

$$
\operatorname{dist} \underset{K(x, y)}{\widetilde{1}}\left(y^{1 / p}, K(x, y)\right) \leq \delta^{-} .
$$

Proof. As in the proof of the previous lemma, is enough to show that $v\left(y^{1 / p}-f\right)<$ $\delta$ for every $f \in K\left[x, \frac{1}{x}, y\right]$. Take an element $f \in K\left[x, \frac{1}{x}, y\right]$ and write it in the form (7). For every $i \in\{-n, \ldots, m\}$ and $j \in\{0, \ldots, l\}$ set

$$
h_{i j}=a_{i j} x^{i} y^{j}=\sum_{\left(m_{1}, \ldots, m_{j}\right) \in \mathbb{N}^{j}} a_{i j} x^{i+\frac{p n_{m_{1}}}{s_{m_{1}}}+\cdots+\frac{p n_{m_{j}}}{s_{m_{j}}} .}
$$

Again we show that for each $j$ there is $N_{j} \in \mathbb{N}$ such that for every $i \in$ $\{-n, \ldots, m\}$ and $N \geq N_{j}$ we have $\frac{n_{N}}{s_{N}} \notin \operatorname{supp} h_{i j}$. Since $y^{1 / p}=\sum_{i=1}^{\infty} x^{\frac{n_{i}}{s_{i}}}$, this means that $\operatorname{supp} h_{i j}$ and supp $y^{1 / p}$ have at most finitely many common elements. As $s_{i}>1$ for every $i \in \mathbb{N}$, we can set $N_{0}=1$. Take now $j \in\{1, \ldots, l\}$ and suppose that

$$
\frac{n_{N}}{s_{N}}=i+\frac{p n_{m_{1}}}{s_{m_{1}}}+\cdots+\frac{p n_{m_{j}}}{s_{m_{j}}}
$$

for some $N \in \mathbb{N}, m_{1}, \ldots, m_{j} \in \mathbb{N}$ and $i \in\{-n, \ldots, m\}$. Since $s_{N}>1$ and $\operatorname{gcd}\left(n_{N}, s_{N}\right)=1$, we deduce that $s_{N}$ divides $s_{m_{1}} \cdot \ldots \cdot s_{m_{j}}$. As the elements $s_{i}$ are pairwise coprime, $s_{N}=s_{m_{i}}$ for some $i \leq j$. Without loss of generality we can assume that exactly $k$ last indices are equal to $N$, that is $m_{j-t} \neq m_{j-k+1}=\ldots=$ $m_{j}=N$ if $k \leq t \leq j-1$.

If $k<j$, then

$$
\frac{n_{N}}{s_{N}}(1-k p)=\frac{d}{s_{m_{1}} \cdot \ldots \cdot s_{m_{j-k}}}
$$


for some integer $d$. Since by assumption $s_{N}$ is coprime with $s_{m_{1}} \cdot \ldots \cdot s_{m_{j-k}}$, this yields that both hand sides of the above equality are nonzero integers. This in particular implies that $s_{N}$ divides $1-k p$, as $\operatorname{gcd}\left(n_{N}, s_{N}\right)=1$.

Assume now that $j=k$. Then

$$
\frac{n_{N}}{s_{N}}(1-k p)=i \text {. }
$$

As in the previous case we deduce that $s_{N}$ divides $1-k p=1-j p$.

Take $N_{j} \in \mathbb{N}$ such that $s_{N_{j}}>j p-1$. Since the sequence $\left(s_{n}\right)_{n \in \mathbb{N}}$ is strictly increasing, for every $N \geq N_{j}$ and $k \in\{1, \ldots, j\}$ we obtain that $s_{N} \geq s_{N_{j}}>k p-1$. Consequently $s_{N}$ does not divide $1-k p$. Thus equality (11) cannot hold and $\frac{n_{N}}{s_{N}} \notin \operatorname{supp} h_{i j}$ for any such $N$. thus

Set $N_{f}=\max \left\{N_{j} \mid 0 \leq j \leq l\right\}$. By what we have shown, $\frac{n_{N_{f}}}{s_{N_{f}}} \notin \operatorname{supp} f$ and

$$
v\left(y^{1 / p}-f\right)=v\left(\sum_{i=1}^{\infty} x^{\frac{n_{i}}{s_{i}}}-f\right)<\frac{n_{N_{f}}}{s_{N_{f}}}<\delta .
$$

Proof of Theorem 4.7:. We take $(K(x) \mid K, v)$ to be the rational function field equipped with the $x$-adic valuation $v$, hence $v$ is trivial on $K$. The field $K(x)$ can be considered as a subfield of the power series field $\left(K\left(\left(x^{\Gamma}\right)\right), v_{x}\right)$ with the canonical valuation $v_{x}$. In the following constructions we choose the element $y \in K\left(\left(x^{\Gamma}\right)\right)$ in such a way that the element is transcendental over $K(x)$ and such that $v K(x, y)=v K\left(\left(x^{\Gamma}\right)\right)=\Gamma$.

Without loss of generality we can assume that $\left(\frac{1}{p^{\infty}} Z\right) \cap \Gamma=\mathbb{Z}$ and $v x=1$. Indeed, if $\left(\frac{1}{p^{\infty}} Z\right) \cap \Gamma=\frac{s}{p^{k}} \mathbb{Z}$ for some nonnegative integer $k$ and a natural number $s$, we replace in our construction $v$ by $\frac{s}{p^{k}} v$.

We construct the element $y$ depending on the group $\Gamma$ and the element $\delta$. In each of the cases we construct a sequence $\left(s_{i}\right)_{i \in \mathbb{N}}$ of positive integers and a sequence $\left(n_{i}\right)_{i \in \mathbb{N}}$ of integers such that

$$
\left\{\begin{array}{l}
\sum_{i=1}^{\infty} \frac{1}{s_{i}} \mathbb{Z}=\Gamma, \\
s_{i} \text { is coprime with } p \text { and } n_{i} \text { for every } i \in \mathbb{N}, \\
\gamma_{i}:=\frac{n_{i}}{s_{i}}, i \in \mathbb{N}, \text { form a strictly increasing sequence converging to } \delta .
\end{array}\right.
$$

Note that then

$$
\mathbb{Z}+\sum_{i=1}^{\infty} p \gamma_{i} \mathbb{Z}=\mathbb{Z}+\sum_{i=1}^{\infty} \frac{1}{s_{i}} \mathbb{Z}=\Gamma .
$$

After a suitable choice of $n_{i}$ and $s_{i}$ we set

$$
y=\sum_{i=1}^{\infty} x^{p \gamma_{i}} \in K\left(\left(x^{\Gamma}\right)\right) .
$$


Take $F:=K(x)^{h}$ and $K(x, y)^{h}$ to be the henselizations of $K(x)$ and $K(x, y)$ inside of $\left(K\left(\left(x^{\Gamma}\right)\right), v\right)$, respectively. If $F(y)^{h}$ denotes the henselization of $F(y)$ inside of $K\left(\left(x^{\Gamma}\right)\right)$, then $F(y)^{h}=K(x, y)^{h}$. Hence, applying Theorem 6.1 of [15] to the henselian field $F$ with $z=y$, we obtain that $x^{p \gamma_{i}} \in K(x, y)^{h}$ for all $i \in \mathbb{N}$. Since $p$ is coprime with the denominator $s_{i}$ of $\gamma_{i}$, we deduce that also $x^{\gamma_{i}} \in K(x, y)^{h}$ for all $i \in \mathbb{N}$. Moreover, as $v F=v K=\mathbb{Z}$, by (13) we have $v F+\sum_{i=1}^{\infty} p \gamma_{i} \mathbb{Z}=\Gamma$. Since $\Gamma$ is non-discrete, the group $\left(v F+\sum_{i=1}^{\infty} p \gamma_{i} \mathbb{Z}\right) / v F$ is infinite. Thus Theorem 6.1 of [15] yields that $v F(y)=\mathbb{Z}+\sum_{i=1}^{\infty} p \gamma_{i} \mathbb{Z}$ and $y$ is transcendental over $F$, thus also over $K(x)$. Since the henselization of a valued field is an immediate extension of the field, we obtain that

$$
v K(x, y)=v K(x, y)^{h}=v F(y)=\Gamma .
$$

Moreover, $K \subseteq K(x, y) v \subseteq K\left(\left(x^{\Gamma}\right)\right) v=K$. Therefore $K(x, y) v=K$.

Consider now the purely inseparable extension $\left(K\left(x, y^{1 / p}\right) \mid K(x, y), v\right)$ of degree $p$. Since

$$
y^{1 / p}=\sum_{i=1}^{\infty} x^{\gamma_{i}} \in K\left(\left(x^{\Gamma}\right)\right)
$$

as in the case of $(K(x, y), v)$ one can show that $v K\left(x, y^{1 / p}\right)=\Gamma$ and $K\left(x, y^{1 / p}\right) v=K$. Thus $\left(K\left(x, y^{1 / p}\right) \mid K(x, y), v\right)$ is an immediate extension.

Denote by $K(x, y)^{c}$ the completion of $(K(x, y), v)$ contained in $\left(K\left(\left(x^{\Gamma}\right)\right), v\right)$. Take a natural number $N$. Since the valuation $v$ is of rank 1 and $x^{\gamma_{i}} \in K(x, y)^{h}$ for all $i$, Theorem 4.4 yields that that the elements $x^{\gamma_{i}}$ lie in the completion of $K(x, y)$. Hence also $\sum_{i=1}^{N} x^{\gamma_{i}} \in K(x, y)^{c}$. Thus there is an element $g_{N} \in K(x, y)$ such that

$$
v\left(\sum_{i=1}^{N} x^{\gamma_{i}}-g_{N}\right) \geq \gamma_{N+1}
$$

Therefore we obtain that

$$
v\left(y^{1 / p}-g_{N}\right)=v\left(\sum_{i=1}^{N} x^{\gamma_{i}}-g_{N}+\sum_{i=N+1}^{\infty} x^{\gamma_{i}}\right) \geq \gamma_{N+1} .
$$

Since $\left(\gamma_{n}\right)_{n \in \mathbb{N}}$ is strictly increasing and converging to $\delta$, we deduce that

$$
\text { dist } \underset{K(x, y)}{\widetilde{1}}\left(y^{1 / p}, K(x, y)\right) \geq \delta^{-} .
$$

To construct the sequences $\left(s_{i}\right)_{i \in \mathbb{N}}$ and $\left(n_{i}\right)_{i \in \mathbb{N}}$, we consider two cases separately.

Case 1. Assume first that there is at least one prime $q$ such that $\frac{1}{q^{\infty}} \mathbb{Z} \subseteq \Gamma$. As $\Gamma$ is not $p$-divisible, $q \neq p$. Take $\left(r_{i}\right)_{i \in \mathbb{N}}$ to be the strictly increasing sequence of all natural numbers $m$ coprime with $q$ such that $\frac{1}{m} \in \Gamma$. Furthermore, we take $\left(e_{i}\right)_{i \in \mathbb{N}}$ 
to be a strictly increasing sequence of natural numbers and set $s_{i}:=r_{i} q^{e_{i}}$. By the choice of the sequences $r_{i}$ and $e_{i}$ we obtain that then

$$
\sum_{i=1}^{\infty} \frac{1}{s_{i}} \mathbb{Z}=\sum_{i=1}^{\infty} \frac{1}{r_{i} q^{e_{i}}} \mathbb{Z}=\Gamma .
$$

Hence first of the conditions (12) holds.

If $\delta=\infty$, choose a sequence of integers $\left(n_{i}\right)_{i \in \mathbb{N}}$ such that $n_{i}$ is coprime with $r_{i} q$ for each $i$ and the sequence

$$
\gamma_{i}=\frac{n_{i}}{s_{i}}=\frac{n_{i}}{r_{i} q^{e_{i}}}, \quad i \in \mathbb{N},
$$

is strictly increasing and unbounded. Since $\gamma_{i} \in \Gamma$, the prime number $p$ does not divide $r_{i} q^{e_{i}}$. Hence $p n_{i}$ is coprime with $s_{i}=r_{i} q^{e_{i}}$ for every $i$. Therefore the sequences of $n_{i}$ and $s_{i}$ satisfy conditions (12). As we have seen, this yields that the element $y$ is transcendental over $K(x)$ and the extension $\left(K\left(x, y^{1 / p}\right) \mid K(x, y), v\right)$ is immediate.

Since $\lim _{n \rightarrow \infty} \gamma_{n}=\infty=\delta$, by inequality (14) we obtain that

$$
\operatorname{dist} \underset{K(x, y)}{\widetilde{1}}\left(y^{1 / p}, K(x, y)\right)=\infty .
$$

Assume now that $\delta \in \mathbb{R}$. We modify our choice of the elements $e_{i}$ and $n_{i}$ in the above construction of the element $y$ to obtain that the sequence $\gamma_{i}=\frac{n_{i}}{r_{i} q^{e_{i}}}$, $i \in \mathbb{N}$, is strictly increasing, convergent to $\delta$ and dist $\widetilde{K(x, y)}\left(y^{1 / p}, K(x, y)\right)=\delta^{-}$. We choose the elements $e_{i}$ and $n_{i}$ by induction on $i$. Take any $e_{1} \in \mathbb{N}$ and set

$$
n_{1}:=\max \left\{k \in \mathbb{Z} \mid \operatorname{gcd}\left(k, q r_{1}\right)=1 \text { and } \frac{k}{q^{e_{1}} r_{1}}<\delta\right\} \text {. }
$$

Define $\gamma_{1}=\frac{n_{1}}{r_{1} q^{e_{1}}}$. Take a natural number $j$. If $j>1$, assume that for every $i \in\{2, \ldots, j\}$ we have chosen $e_{i}$ and $n_{i}$ in such a way that for $\gamma_{i}=\frac{n_{i}}{r_{i} q^{e_{i}}}$ we have

(i) $\gamma_{i-1}<\gamma_{i}<\delta$,

(ii) $\delta-\gamma_{i}<\frac{1}{i}$,

(iii) $e_{i}-e_{i-1} \geq i-1$,

(iv) $\operatorname{gcd}\left(n_{i}, r_{i} q\right)=1$.

Note that the choice of $n_{1}$ and condition (iv) implies that $n_{i} \neq 0$ for $i \leq j$. Take an integer $k$ and a natural number $t$ such that $\gamma_{j}<\frac{k}{q^{t}}<\delta$ and

$$
\delta-\frac{k}{q^{t}}<\frac{1}{j+1} .
$$

Such numbers $k$ and $t$ exist as $\frac{1}{q^{\infty}} \mathbb{Z}$ is dense in $\mathbb{Q}$, hence in $\mathbb{R}$. Take now $e_{j+1} \in \mathbb{N}$ such that $e_{j+1} \geq \max \left\{t+1, e_{j}+j\right\}$ and

$$
\gamma_{j}<\frac{k}{q^{t}}+\frac{1}{r_{j+1} q^{e_{j+1}}}<\delta .
$$


Set $n_{j+1}=k r_{j+1} q^{e_{j+1}-t}+1$ and $\gamma_{j+1}=\frac{n_{j+1}}{r_{j+1} q^{e} j+1}=\frac{k}{q^{t}}+\frac{1}{r_{j+1} q^{e} j+1}$. Directly from the choice of $\gamma_{j+1}$ it follows that conditions (i), (iii) and (iv) hold for $i=j+1$. Moreover, by the choice of $n_{j+1}$ we have

$$
\delta-\gamma_{j+1}=\delta-\left(\frac{k}{q^{t}}+\frac{1}{r_{j+1} q^{e_{j+1}}}-\right)=\left(\delta-\frac{k}{q^{t}}\right)-\frac{1}{r_{j+1} q^{e_{j+1}}}<\frac{1}{j+1} .
$$

Note that $\delta-\gamma_{i} \leq \frac{1}{i}$ for all $i \geq 2$, whence $\lim _{i \rightarrow \infty} \gamma_{i}=\delta$. Hence the sequences $n_{i}$ and $s_{i}:=r_{i} q^{e_{i}}, i \in \mathbb{N}$, satisfy conditions (12). Again, this yields that the element $y$ is transcendental over $K(x)$ and $\left(K\left(x, y^{1 / p}\right) \mid K(x, y), v\right)$ is an immediate extension.

Furthermore, by inequality (14) we obtain that dist $\widetilde{K(x, y)}\left(y^{1 / p}, K(x, y)\right) \geq$ $\delta^{-}$. Together with Lemma 4.8 this shows that

$$
\operatorname{dist} \underset{K(x, y)}{\widetilde{1}}\left(y^{1 / p}, K(x, y)\right)=\delta^{-} .
$$

Case 2. Assume now that there is no prime $q$ such that $\frac{1}{q^{\infty}} \mathbb{Z} \subseteq \Gamma$. Hence, for every prime $q \neq p$ there is a nonnegative integer $N_{q}$ such that $q^{-N_{q}} \in \Gamma$ and $q^{-N_{q}-1} \notin \Gamma$. Since $\Gamma$ is non-discrete, there are infinitely many primes $q$ such that $N_{q} \geq 1$. Take $\left(r_{i}\right)_{i \in \mathbb{N}}$ to be the strictly increasing sequence obtained from all the elements $q^{N_{q}}$ with $N_{q} \geq 1$. Note that by our assumptions $N_{p}=0$, hence $p$ does not divide $r_{i}, i \in \mathbb{N}$.

If $\delta=\infty$, take $s_{i}=r_{i}$ for all $i \in \mathbb{N}$. Then $\sum_{i=1}^{\infty} \frac{1}{s_{i}} \mathbb{Z}=\Gamma$. Take $\left(n_{i}\right)_{i \in \mathbb{N}}$ to be any sequence of integers such that $\operatorname{gcd}\left(n_{i}, s_{i}\right)=1$ for every $i \in \mathbb{N}$ and the sequence $\gamma_{i}=\frac{n_{i}}{s_{i}}, i \in \mathbb{N}$ is strictly increasing and unbounded. Since $p$ does not divide $r_{i}=s_{i}$ for $i \in \mathbb{N}$, the sequences $\left(s_{i}\right)$ and $\left(n_{i}\right)$ satisfy conditions (12). This yields that the element $y$ is transcendental over $K(x)$ and the extension $\left(K\left(x, y^{1 / p}\right) \mid K(x, y), v\right)$ is immediate.

Since $\lim _{n \rightarrow \infty} \gamma_{n}=\infty=\delta$, by inequality (14) we obtain that

$$
\text { dist } \widetilde{K(x, y)}\left(y^{1 / p}, K(x, y)\right)=\infty .
$$

Suppose now that $\delta \in \mathbb{R}$. We construct the sequences $n_{i}$ and $s_{i}$ by induction on $i$. If $N_{2}=0$, set $s_{1}=r_{1}$. Otherwise, if $m_{1} \in \mathbb{N}$ is such that $r_{m_{1}}$ is a power of 2 , then take $s_{1}=r_{1} \cdot \ldots \cdot r_{m_{1}}$. Set

$$
n_{1}:=\max \left\{k \in \mathbb{Z} \mid \operatorname{gcd}\left(k, s_{1}\right)=1 \text { and } \frac{k}{s_{1}}<\delta\right\} \text {. }
$$

Take a natural number $j$. If $j>1$, assume that for every $i \in\{2, \ldots, j\}$ we have chosen elements $m_{i} \in \mathbb{N}$ and $n_{i} \in \mathbb{Z}$ in such a way that for $s_{i}:=r_{m_{i-1}+1} \cdot \ldots \cdot r_{m_{i}}$ and $\delta_{i}=\frac{n_{i}}{s_{i}}$ we have

(i') $m_{i-1}<m_{i}$,

(ii') $\gamma_{i-1}<\gamma_{i}<\delta$,

(iii') $\operatorname{gcd}\left(n_{i}, s_{i}\right)=1$, 
(iv') $\delta-\gamma_{i}<\frac{1}{i}$.

Since $\left(r_{i}\right)_{i \in \mathbb{N}}$ is a strictly increasing sequence of natural numbers, there is a natural number $m_{j+1}>m_{j}$ and $k \in \mathbb{Z}$ such that

$$
\begin{gathered}
\gamma_{j}<\frac{k}{r_{m_{j+1}}}<\frac{k+2}{r_{m_{j+1}}}<\delta, \\
\delta-\frac{k}{r_{m_{j+1}}}<\frac{1}{j+1} .
\end{gathered}
$$

Then for $s_{j+1}:=r_{m_{j}+1} \cdot \ldots \cdot r_{m_{j+1}}$ by (15) we obtain that

$$
\gamma_{j}<\frac{k}{r_{m_{j+1}}}<\frac{k}{r_{m_{j+1}}}+\frac{1}{s_{j+1}}<\frac{k}{r_{m_{j+1}}}+\frac{2}{s_{j+1}}<\frac{k+2}{r_{m_{j+1}}}<\delta .
$$

As $r_{m_{j+1}}$ is a power of a prime, at least one of the numbers $k r_{m_{j}+1} \cdot \ldots \cdot r_{m_{j+1}-1}+1$ and $k r_{m_{j}+1} \ldots \cdot r_{m_{j+1}-1}+2$ is coprime with $r_{m_{j+1}}$. Choose such a number and denote it by $n_{j+1}$. As $j \geq 2$, the numbers $r_{m_{j}+1}, \ldots, r_{m_{j+1}-1}$ are powers of odd primes, and thus $n_{j+1}$ is coprime with all these numbers. Consequently, $\operatorname{gcd}\left(s_{j+1}, n_{j+1}\right)=1$. Hence equation (iii') holds for $i=j+1$. Set $\gamma_{j+1}:=\frac{n_{j+1}}{s_{j+1}}$. Then (17) together with the choice of $n_{j+1}$ yields that $\gamma_{j}<\gamma_{j+1}<\delta$. It remains to show inequality (iv'). By inequality (16)

$$
\begin{aligned}
\delta-\gamma_{j+1} & \leq \delta-\frac{k r_{m_{j}+1} \cdot \ldots \cdot r_{m_{j+1}-1}+1}{s_{j+1}}=\delta-\frac{k}{r_{m_{j+1}}}-\frac{1}{r_{m_{j}+1} \cdot \ldots \cdot r_{m_{j+1}}} \\
& \leq \frac{1}{j+1}-\frac{1}{r_{m_{j}+1} \cdot \ldots \cdot r_{m_{j+1}}}<\frac{1}{j+1} .
\end{aligned}
$$

Note that the sequences $\left(n_{i}\right)_{i \in \mathbb{N}}$ and $\left(s_{i}\right)_{i \in \mathbb{N}}$ satisfy conditions (12). Indeed, by the choice of the elements $s_{i}$ and $r_{i}$

$$
\sum_{i=1}^{\infty} \frac{1}{s_{i}} \mathbb{Z}=\sum_{i=1}^{\infty} \frac{1}{r_{i}} \mathbb{Z}=\Gamma .
$$

By assumption, $p$ does not divide any element $r_{i}$. This together with equation (iii') yields that $\operatorname{gcd}\left(p n_{i}, s_{i}\right)=1$ for every $i \in \mathbb{N}$. Inequalities (ii') and (iv') imply that $\left(\gamma_{i}\right)_{i \in \mathbb{N}}$ is a strictly increasing sequence converging to $\delta$. As in the previous cases, this yields that the element $y$ is transcendental over $K(x)$ and the extension $\left(K\left(x, y^{1 / p}\right) \mid K(x, y), v\right)$ is immediate.

Moreover, by inequality (14) we obtain that dist $\widetilde{K(x, y)}\left(y^{1 / p}, K(x, y)\right) \geq \delta^{-}$. Together with Lemma 4.9 this yields that dist $\widetilde{K(x, y)}\left(y^{1 / p}, K(x, y)\right)=\delta^{-}$.

The above constructions enable us to obtain Artin-Schreier defect extensions of rational function fields of a given distance. 
Corollary 4.10. Take a field $K$ of positive characteristic p, a non-discrete subgroup $\Gamma$ of $\mathbb{Q}$ which is not $p$-divisible and a negative real number $\delta$. Then there is a valuation $v$ on the rational function field $K(x, y) \mid K$ whose restriction to $K$ is trivial with $v K(x, y)=\Gamma$, and such that a root $\vartheta$ of the polynomial $X^{p}-X-y$ generates an Artin-Schreier defect extension $(K(x, y, \vartheta) \mid K(x, y), v)$ of distance $\delta^{-}$.

Proof. As in the proof of Theorem 4.7 we construct the element $y \in K\left(\left(x^{\Gamma}\right)\right)$ such that the field $K(x, y)$ together with the restriction $v$ of the canonical valuation of $K\left(\left(x^{\Gamma}\right)\right)$ admits an immediate purely inseparable extension $\left(K\left(x, y^{1 / p}\right), v\right)$ with dist $\widetilde{K(x, y)}\left(y^{1 / p}, K(x, y)\right)=\delta^{-}$. We show now that since $\delta<0$, we can construct the element $y$ in such a way that

$$
v y^{\frac{1}{p}}=\gamma_{1}>p \delta .
$$

In Case 1 we take an integer $k$ and a natural number $t$ such that $p \delta<\frac{k}{q^{t}}<\delta$. Further we take a natural number $e_{1} \geq t+1$ such that

$$
p \delta<\frac{k}{q^{t}}+\frac{1}{r_{1} q^{e_{1}}}<\delta .
$$

Then $n_{1}:=k r_{1} q^{e_{1}-t}+1$ is coprime with $r_{1} q$ and for $\gamma_{1}=\frac{n_{1}}{r_{1} q^{e_{1}}}=\frac{k}{q^{t}}+\frac{1}{r_{1} q_{e_{1}}}$ we have $p \delta<\gamma_{1}<\delta$.

In Case 2 take a natural number $m$ and an integer $k$ such that

$$
p \delta<\frac{k}{r_{m}}<\frac{k+2}{r_{m}}<\delta .
$$

Set $m_{1}=\max \left\{m, i \mid 2\right.$ divides $\left.r_{i}\right\}$ and $s_{1}=r_{1} \cdot \ldots \cdot r_{m_{1}}$. We then construct $n_{1}$ as in the induction step of case 2 to make sure that $\operatorname{gcd}\left(n_{1}, s_{1}\right)=1$ and $\frac{k}{r_{m}}<\frac{n_{1}}{s_{1}}<\delta$. For $\gamma_{1}=\frac{n_{1}}{s_{1}}$ we obtain that $p \delta<\gamma_{1}<\delta$.

Consider the polynomial $Y^{p}-Y-y$. Note that from (18) we obtain that

$$
(p-1) v 1=0>p \delta^{-}-\gamma_{1}=p \text { dist } \widetilde{K(x, y)}\left(y^{1 / p}, K(x, y)\right)-\frac{v y}{p} .
$$

Now Theorem 4.5 of [17] yields that a root $\vartheta$ of the polynomial $X^{p}-X-y$ generates a dependent Artin-Schreier defect extension of $K(x, y)$. Moreover, $\vartheta \approx_{K} y^{1 / p}$. Thus,

$$
\text { dist } \widetilde{K(x, y)}(\vartheta, K(x, y))=\operatorname{dist} \underset{K(x, y)}{\widetilde{1}}\left(y^{1 / p}, K(x, y)\right)=\delta^{-} .
$$

Open Access This article is distributed under the terms of the Creative Commons Attribution 4.0 International License (http://creativecommons.org/licenses/by/4.0/), which permits unrestricted use, distribution, and reproduction in any medium, provided you give appropriate credit to the original author(s) and the source, provide a link to the Creative Commons license, and indicate if changes were made. 


\section{References}

[1] Blaszczok, A.: On the structure of immediate extensions of valued fields, Ph.D. thesis, University of Silesia (2014)

[2] Blaszczok, A.: Infinite towers of Artin-Schreier defect extensions of rational function fields. In: Second International Conference and Workshop on Valuation Theory (Segovia/El Escorial, Spain, 2011), EMS Series of Congress Reports, vol. 10, pp. 16-54 (2014)

[3] Blaszczok, A., Kuhlmann, F.-V.: Algebraic independence of elements in immediate extensions of valued fields. J. Algebra 425, 179-214 (2015)

[4] Blaszczok, A., Kuhlmann, F.-V.: Corrections and notes to "Value groups, residue fields and bad places of rational function fields". Trans. Am. Math. Soc. 3675, 4505-4515 (2015)

[5] Blaszczok, A., Kuhlmann, F.-V.: On maximal immediate extensions of valued fields. Math. Nachr. 290, 7-18 (2017)

[6] Blaszczok, A., Kuhlmann, F.-V.: Counting of the number of distinct distances of elements in valued field extensions. J. Algebra 509, 192-211 (2018)

[7] Cutkosky, S.D., Piltant, O.: Ramifcation of valuations. Adv. Math. 183, 1-79 (2004)

[8] Endler, O.: Valuation Theory. Springer, Berlin (1972)

[9] Engler, A.J., Prestel, A.: Valued Fields. Springer Monographs in Mathematics. Springer, Berlin (2005)

[10] Hahn, H.: Über die nichtarchimedischen Größensysteme. S.-B. Akad. Wiss. Wien Math.-naturw. Kl. Abt. IIa 116, 601-655 (1907)

[11] Kaplansky, I.: Maximal fields with valuations I. Duke Math. J. 9, 303-321 (1942)

[12] Karpilovsky, G.: Topics in Field Theory. Mathematics Studies, vol. 155. North Holland, Amsterdam (1989)

[13] Khanduja, S.K., Singh, A.P.: On theorem of Tignol for defectless extensions and its converse. J. Algebra 288, 400-408 (2005)

[14] Krull, W.: Allgemeine bewertungstheorie. J. Reine Angew. Math. 167, 160-196 (1932)

[15] Kuhlmann, F.-V.: Value groups, residue fields and bad places of rational function fields. Trans. Am. Math. Soc. 356, 4559-4600 (2004)

[16] Kuhlmann, F.-V.: Additive polynomials and their role in the model theory of valued fields. In: Logic in Tehran, Proceedings of the Workshop and Conference on Logic, Algebra, and Arithmetic, held October 18-22, 2003. Lecture Notes in Logic, vol. 26, pp. 160-203 (2006)

[17] Kuhlmann, F.-V.: A classification of Artin-Schreier defect extensions and a characterization of defectless fields. Ill. J. Math. 54, 397-448 (2010)

[18] Kuhlmann, F.-V.: Approximation of elements in henselizations. Manuscr. Math. 136, 461-474 (2011)

[19] Kuhlmann, F.-V.: Defect. In: Fontana, M., Kabbaj, S.-E., Olberding, B., Swanson, I. (eds.) Commutative Algebra-Noetherian and non-Noetherian Perspectives, pp. 277318. Springer, Berlin (2011)

[20] Kuhlmann, F.-V.: The model theory of tame valued fields. J. Reine Angew. Math. 719, $1-43(2016)$

[21] Kuhlmann, F.-V.: Valuation Theory, Book in Preparation. http://math.usask.ca/ fvk/ Fvkbook.htm

[22] Kuhlmann, F.-V. and Piltant, O., Higher ramification groups for Artin-Schreier defect extensions. (in preparation)

[23] Kuhlmann, F.-V., Vlahu, I.: The relative approximation degree in valued function fields. Math. Z. 276, 203-235 (2014) 
[24] Lang, S.: Algebra Graduate Texts in Mathematics. Springer, New York (2002)

[25] MacLane, S., Schilling, O.F.G.: Zero-dimensional branches of rank 1 on algebraic varieties. Ann. Math. 40, 507-520 (1939)

[26] Warner, S.: Topological Fields. Mathematics Studies, vol. 157. North Holland, Amsterdam (1989)

[27] Zariski, O., Samuel, P.: Commutative Algebra. vol. II. New York (1960)

Publisher's Note Springer Nature remains neutral with regard to jurisdictional claims in published maps and institutional affiliations. 